Piermarco Cannarsa $\cdot$ Pierre Cardaliaguet

\title{
Representation of equilibrium solutions to the table problem for growing sandpiles
}

Received April 23, 2003 and in revised form December 11, 2003

\begin{abstract}
In the dynamical theory of granular matter the so-called table problem consists in studying the evolution of a heap of matter poured continuously onto a bounded domain $\Omega \subset \mathbb{R}^{2}$. The mathematical description of the table problem, at an equilibrium configuration, can be reduced to a boundary value problem for a system of partial differential equations. The analysis of such a system, also connected with other mathematical models such as the Monge-Kantorovich problem, is the object of this paper. Our main result is an integral representation formula for the solution, in terms of the boundary curvature and of the normal distance to the cut locus of $\Omega$.
\end{abstract}

Keywords. Granular matter, eikonal equation, singularities, semiconcave functions, viscosity solutions, optimal mass transfer

\section{Introduction}

In recent years, the attention of many authors has been focussed on the system of partial differential equations

$$
\begin{cases}-\operatorname{div}(v D u)=f & \text { in } \Omega \\ |D u|-1=0 & \text { in }\{v>0\}\end{cases}
$$

in a given domain $\Omega \subset \mathbb{R}^{n}$.

For instance, system (1) arises in Monge-Kantorovich theory as necessary conditions to be satisfied by an optimal mass transfer plan (see [16], [2] and [20]). In a related framework, system (1) characterizes the limit, as $p \rightarrow \infty$, of the $p$-Laplace equation $-\operatorname{div}\left(|D u|^{p-2} D u\right)=f($ see [11], [24], [6] and [15]). Furthermore, the above system has been applied to an idealized model for compression molding in [5], and to shape optimization in [9].

Work supported in part by the European Community's Human Potential Programme under contract HPRN-CT-2002-00281 [Evolution Equations].

P. Cannarsa: Dipartimento di Matematica, Università di Roma Tor Vergata, Via della Ricerca Scientifica 1, 00133 Roma, Italy; e-mail: cannarsa@axp.mat.uniroma2.it

P. Cardaliaguet: Université de Bretagne Occidentale, UFR des Sciences et Techniques, 6 Av. Le Gorgeu, BP 809, 29285 Brest, France; e-mail: Pierre.Cardaliaguet@univ-brest.fr

Mathematics Subject Classification (2000): Primary 35C15; Secondary 35A21, 35Q99, 90 B06 
Another interesting example of application of (1) stems from granular matter theory (see [4], [27], [6] and [15]). Recently, Hadeler and Kuttler [22] proposed a new model to study the evolution of a sandpile created by pouring dry matter onto a 'table'. In such a model, built on previous work by Boutreux and de Gennes [10], the table is represented by a bounded domain $\Omega \subset \mathbb{R}^{2}$, and the matter source by a function $f(t, x) \geq 0$. The physical description of the growing heap is based on the introduction of the so-called standing and rolling layers. The former collects the amount of matter that remains at rest, the latter represents matter moving down along the surface of the standing layereventually falling down when the base of the heap touches the boundary of the table.

As pointed out in [22], system [1] is related to equilibrium configurations that may occur in physical models with a constant source. To explain this connection, let us denote by $u(x)$ and $v(x)$, respectively, the heights of the standing and rolling layers at a point $x \in \Omega$, for an equilibrium configuration. For physical reasons, the slope of the standing layer cannot exceed a given constant - typical of the matter under consideration - that we normalize to 1 . Consequently, the standing layer must vanish on the boundary of the table. So, $|D u| \leq 1$ in $\Omega$ and $u=0$ on $\partial \Omega$. Also, in the region where $v$ is positive, the standing layer has to be 'maximal', for otherwise more matter would roll down there to rest. On the other hand, the rolling layer results from transporting matter, poured by the source, along the surface of the standing layer at a speed that is assumed proportional to the slope $D u$, with constant equal to 1 . The above considerations lead to the boundary value problem

$$
\begin{cases}-\operatorname{div}(v D u)=f & \text { in } \Omega, \\ |D u|-1=0 & \text { in }\{v>0\}, \\ |D u| \leq 1, \quad u, v \geq 0 & \text { in } \Omega, \\ u=0 & \text { on } \partial \Omega .\end{cases}
$$

For the reader who is interested in Monge-Kantorovich theory we note that a connection of the above system with such a theory could be obtained by looking at the so-called dual problem of maximizing

$$
\int_{\Omega} u(x) f(x) d x
$$

over all Lipschitz continuous functions $u: \Omega \rightarrow \mathbb{R}$, with $\operatorname{Lip}(u) \leq 1$, vanishing on $\partial \Omega$. Indeed, as proved in [8], the boundary value problem (2) turns out to be the system of necessary conditions satisfied by any maximizer $u$ of (3), with $v$ equal to the associated Lagrange multiplier. Such a framework is also related to the optimization problem studied in [12].

The main purpose of the present work is to provide a full analysis of problem (2), including existence, uniqueness, and representation of the solution. To describe our results more precisely, let us denote by $d: \bar{\Omega} \rightarrow \mathbb{R}$ the distance function from the boundary of $\Omega$ and by $\Sigma$ the singular set of $d$, that is, the set of points $x \in \Omega$ at which $d$ is not differentiable. The closure of that set is also called the cut locus of $\partial \Omega$ in $\Omega$. If we introduce the projection $\Pi(x)$ of $x$ onto $\partial \Omega$ in the usual way, then $\Sigma$ is also the set of points $x$ at which $\Pi(x)$ is not a singleton. Since $d$ is Lipschitz continuous, $\Sigma$ has Lebesgue 
measure zero. In our analysis, a major role will be played by the normal distance to $\bar{\Sigma}$, that is, the function

$$
\tau(x)=\min \{t \geq 0: x+t D d(x) \in \bar{\Sigma}\} \quad \forall x \in \bar{\Omega} \backslash \bar{\Sigma} .
$$

It is well known that the eikonal equation $|D u|=1$ does not have global smooth solutions in general, and neither does the conservation law $-\operatorname{div}(v D u)=f$. Thus, before stating our main result, we must explain what we mean by a solution of (2). We say that a pair $(u, v)$ of continuous functions in $\Omega$ is a solution of problem (2) if

- $u=0$ on $\partial \Omega,\|D u\|_{\infty, \Omega} \leq 1$, and $u$ is a viscosity solution of

$$
|D u|=1 \quad \text { in }\{x \in \Omega: v(x)>0\},
$$

- $v \geq 0$ in $\Omega$ and, for every test function $\phi \in \mathcal{C}_{\mathrm{c}}^{\infty}(\Omega)$,

$$
\int_{\Omega} v(x)\langle D u(x), D \phi(x)\rangle d x=\int_{\Omega} f(x) \phi(x) d x .
$$

Notice that the maximality of the standing layer, justified above by physical considerations, is now ensured by typical properties of viscosity solutions.

Our main result is the following.

Theorem 1.1. Let $\Omega \subset \mathbb{R}^{2}$ be a bounded domain with boundary of class $\mathcal{C}^{2}$ and $f \geq 0$ be a continuous function in $\Omega$. Then a solution of system (2) is given by the pair $\left(d, v^{f}\right)$, where $v^{f}=0$ on $\bar{\Sigma}$ and

$$
v^{f}(x)=\int_{0}^{\tau(x)} f(x+t D d(x)) \frac{1-(d(x)+t) \kappa(x)}{1-d(x) \kappa(x)} d t \quad \forall x \in \Omega \backslash \bar{\Sigma} .
$$

Here, $\kappa(x)$ denotes the curvature of $\partial \Omega$ at the point $\Pi(x)$.

Moreover, the above solution is unique in the following sense: if $(u, v)$ is another solution of 2$\}$, then $v=v^{f}$ in $\Omega$, and $u=d$ in $\left\{x \in \Omega: v^{f}>0\right\}$.

In the proof of the above theorem, we will first show that the pair $\left(d, v^{f}\right)$ is indeed a solution of the boundary value problem. This will also provide an existence result for (2). Incidentally, recalling the connection of (2) with the maximization problem (3), we note that an existence result for the boundary value problem in question, in any space dimension, could be derived from the results of [8, 9] (see also [16] and [2]). Then we will show that the solution of (2) is unique. We recall that uniqueness results for system (1), with Neumann boundary conditions for $u$, are known in the literature (see [2] and [20]). However, to our knowledge, the boundary value problem (2) has never been addressed explicitly.

As for formula (4), we note that it extends to dimension 2 the representation formula obtained in [22] for the one-dimensional case. The structure of such a formula can be justified by straightforward heuristic arguments that we will sketch in Section 3. Similar heuristic arguments were developed in [15] (see also [6] and [5]) to study a model for sandpile collapse. For such a model, where the source term $f$ is replaced by an expression involving the sandpile height and its time derivative, the evolution of the sandpile base is 
described by means of a representation formula containing curvature terms. A rigorous derivation of such a formula for measurable data is given in [18] using a technique that is completely different from ours-our solution is continuous in $\Omega$ and smooth in $\Omega \backslash \bar{\Sigma}$ and then adapted to sandpile growth around an obstacle in [19].

The main technical tools used in this paper are the results of [3] and [1] that describe the propagation of singularities of semiconcave functions. We also need a Lipschitz regularity result for the normal distance to $\bar{\Sigma}$ proved in [23] (see also [25]). In the Appendix, for the reader's convenience, we provide a simple proof of this result in the 2-dimensional case.

As recalled above, a noteworthy aspect of our result is that we do construct a continuous solution $v^{f}$, instead of just a measure or a function in $L^{1}(\Omega)$. So, Theorem 1.1 could also be viewed as a regularity result. Moreover, formula (4) can be used to derive further regularity properties. In fact, as we will show in a forthcoming paper, $v^{f}$ is Hölder continuous in $\Omega$ if $f$ and $\partial \Omega$ are sufficiently smooth. This result turns out to be optimal.

Except for the Appendix, the paper is entirely devoted to the proof of Theorem 1.1 preliminary properties of the distance function are collected in Section 2, the proof that the pair $\left(d, v^{f}\right)$ is a solution of 2) is given in Section 3, uniqueness is shown in Section 4.

\section{Preliminaries}

In this section we collect our notations and some properties of the distance function. For any $s \in \mathbb{R}$, we set $[s]_{+}=\max \{s, 0\}$ and $[s]_{-}=\max \{-s, 0\}$. We denote by $\langle\cdot, \cdot\rangle$ and $|\cdot|$ the Euclidean scalar product and norm in $\mathbb{R}^{2}$, respectively. For any $x \in \mathbb{R}^{2}$ and $r>0$, $B_{r}(x)$ stands for the open ball with centre $x$ and radius $r$. For any pair $x, y \in \mathbb{R}^{2}$ we denote by $] x, y[$ and $[x, y]$, respectively, the open and closed line segment with extreme points $x$ and $y$.

For any given set $K \subset \mathbb{R}^{2}$ we define $\operatorname{diam} K=\sup \{|y-x|: x, y \in K\}$. If $K \subset \mathbb{R}^{2}$ is closed, we set, for any point $x \in \mathbb{R}^{2}$,

$$
d_{K}(x)=\min _{y \in K}|y-x|, \quad \Pi_{K}(x)=\left\{y \in K: d_{K}(x)=|y-x|\right\} .
$$

For any measurable set $A \subset \mathbb{R}^{2}$, we denote by $|A|$ the Lebesgue measure of $A$. If $u: A \rightarrow \mathbb{R}$ is a bounded measurable function, then $\|u\|_{\infty, A}$ stands for the essential supremum of $u$ in $A$. If $A$ is open and $u$ is Lipschitz continuous, then, by Rademacher's Theorem, $u$ is differentiable a.e. in $A$. In this case, we denote by $\|D u\|_{\infty, A}$ the number $\sup \{|D u(x)|: x \in A, D u(x)$ exists $\}$, and by $D^{*} u(x)$ the set of limiting gradients of $u$ at $x$ defined as

$$
D^{*} u(x)=\left\{\lim _{n} D u\left(x_{n}\right): A \ni x_{n} \rightarrow x, D u\left(x_{n}\right) \text { exists }\right\} .
$$

As usual, the superdifferential of $u$ at a point $x \in A$ is the set

$$
D^{+} u(x)=\left\{p \in \mathbb{R}^{n}: \limsup _{h \rightarrow 0} \frac{u(x+h)-u(x)-\langle p, h\rangle}{|h|} \leq 0\right\},
$$

while the subdifferential $D^{-} u$ is given by the formula $D^{-} u(x)=-D^{+}(-u)(x)$. 
Definition 2.1. We say that $u$ is a viscosity solution of the eikonal equation $|D u|=1$ in an open set $\Omega \subset \mathbb{R}^{2}$ if, for any $x \in \Omega \subset \mathbb{R}^{2}$, we have

$$
\begin{aligned}
& p \in D^{-} u(x) \Rightarrow|p| \geq 1, \\
& p \in D^{+} u(x) \Rightarrow|p| \leq 1 .
\end{aligned}
$$

The results we give below are standard for the eikonal equation. For their proof, we refer the reader to [7], and precisely to Theorem 5.9 and Remark 5.10 in Chapter II there, as well as to Proposition 3.12 in Chapter IV.

Proposition 2.2. Let u be a Lipschitz continuous viscosity solution of the eikonal equation $|D u|=1$ in $\Omega$. Then

(a) For every $x \in \Omega$,

$$
u(x)=\min _{y \in \partial \Omega}\{u(y)+|y-x|\}=\min _{y \in \partial \Omega,] x, y[\subset \Omega}\{u(y)+|y-x|\} .
$$

(b) Let $x \in \Omega$ and let $y \in \partial \Omega$ be such that $u(x)=u(y)+|x-y|$. Then, for any $z \in] x, y[, u$ is differentiable at $z$ and $D u(z)=(x-y) /|x-y|$.

(c) Let $u$ be differentiable at a point $x \in \Omega$ and set

$$
\begin{array}{r}
\bar{t}=\inf \{t>0: x-t D u(x) \notin \Omega\} . \\
\text { Then } y:=x-\bar{t} D u(x) \in \partial \Omega \text { and } u(x)=u(y)+|x-y| .
\end{array}
$$

Remark 2.3. The representation formula in (a) implies, as is well known, that $u$ is locally semiconcave in $\Omega$, i.e., for any convex set $\Omega^{\prime} \subset \subset \Omega$ there is a constant $C \in \mathbb{R}$ such that $x \mapsto u(x)-C|x|^{2}$ is concave in $\Omega^{\prime}$. Consequently,

$$
D^{+} u(x)=\operatorname{co} D^{*} u(x) \quad \forall x \in \Omega
$$

and the set-valued map $x \mapsto D^{+} u(x)$ is upper semicontinuous in $\Omega$, that is, for every $x \in \Omega$,

$$
\Omega \ni x_{n} \rightarrow x, \quad D^{+} u\left(x_{n}\right) \ni p_{n} \rightarrow p \quad(n \rightarrow \infty) \quad \Rightarrow \quad p \in D^{+} u(x) .
$$

Moreover, owing to the structural properties of maximal monotone mappings in Euclidean spaces (see for instance [28, Corollary 12.66]), the set of points where $D u$ itself fails to be differentiable is negligible.

Throughout the paper we assume that

$\Omega$ is a connected bounded open subset of $\mathbb{R}^{2}$ with $\mathcal{C}^{2}$ boundary.

For simplicity, we will write $d$ for $d_{\partial \Omega}$ and $\Pi$ for $\Pi_{\partial \Omega}$. Whenever $x$ has a unique projection onto $\partial \Omega$, with a minor abuse of notation, we will identify the set $\Pi(x)$ with its unique element. 
Remark 2.4. We recall that the distance function $d$ is the unique viscosity solution of the eikonal equation $|D u|=1$ in $\Omega$, with boundary condition $u=0$ in $\partial \Omega$. Equivalently, $d$ is the largest function such that $\|D u\|_{\infty, \Omega} \leq 1$ and $u=0$ on $\partial \Omega$.

We will denote by $\Sigma$ the singular set of the distance from $\partial \Omega$, or briefly, the singular set of $\Omega$, that is, the set of all points $x \in \Omega$ at which $d$ fails to be differentiable. Equivalently, $\Sigma$ is also the set of points $x$ at which $\Pi(x)$ is not a singleton. Since $d$ is Lipschitz continuous, $\Sigma$ has Lebesgue measure 0 .

A standard-yet important-consequence of assumption $\sqrt{6}$ is that $d$ is $\mathcal{C}^{2}$ in $\bar{\Omega} \backslash \bar{\Sigma}$. The result we recall below can be proved using the classical method of characteristics.

Proposition 2.5. Let $x \in \bar{\Omega} \backslash \bar{\Sigma}$ and let $t>0$ be such that $x+s \operatorname{Dd}(x) \notin \bar{\Sigma}$ for every $s \in[0, t)$. Then, for every $s \in[0, t)$,
(a) $d(x+s D d(x))=d(x)+s$,
(b) $\operatorname{Dd}(x+s \operatorname{Dd}(x))=\operatorname{Dd}(x)$,
(c) $\Pi(x+s D d(x))=\Pi(x)$.

In view of items (a) or (c) in the above proposition, $x+s D d(x) \in \bar{\Sigma}$ for some $s>0$. So, the map $\tau: \bar{\Omega} \rightarrow[0, \operatorname{diam} \Omega / 2]$ given by

$$
\tau(x)= \begin{cases}\min \{t \geq 0: x+t \operatorname{Dd}(x) \in \bar{\Sigma}\} & \forall x \in \bar{\Omega} \backslash \bar{\Sigma}, \\ 0 & \forall x \in \bar{\Sigma},\end{cases}
$$

is well defined. Such a map measures the distance of a point $x$ to the set $\bar{\Sigma}$ along the direction of $\operatorname{Dd}(x)$ (which differs from the distance of $x$ to $\bar{\Sigma}$, in general). In this paper, it will be called the normal distance to $\bar{\Sigma}$. In the literature, $\tau$ is often referred to as the distance to the cut locus of $\partial \Omega$.

Hereafter, for any $x \in \partial \Omega$, we denote by $\kappa(x)$ the curvature of $\partial \Omega$ at $x$, with the sign convention that $\kappa \geq 0$ if $\Omega$ is convex. Also, we will label in the same way the extension of $\kappa$ to $\bar{\Omega} \backslash \Sigma$ given by

$$
\kappa(x)=\kappa(\Pi(x)) \quad \forall x \in \bar{\Omega} \backslash \Sigma .
$$

In the result below, $p \otimes q$ stands for the tensor product of two vectors $p, q \in \mathbb{R}^{2}$, defined as $(p \otimes q)(x)=p\langle q, x\rangle, \forall x \in \mathbb{R}^{2}$.

Proposition 2.6. For any $x \in \bar{\Omega}$ and any $y \in \Pi(x)$ we have $\kappa(y) d(x) \leq 1$. If, in addition, $x \in \bar{\Omega} \backslash \bar{\Sigma}$, then

$$
\kappa(x) d(x)<1, \quad D^{2} d(x)=-\frac{\kappa(x)}{1-\kappa(x) d(x)} q \otimes q,
$$

where $q$ is any unit vector such that $\langle q, D d(x)\rangle=0$.

Remark 2.7. Owing to assumption (6), we have

$$
\sup _{y, z \in \partial \Omega, y \neq z} \frac{|D d(z)-D d(y)|}{|z-y|}<\infty .
$$

So, in view of Proposition 2.6, we will denote the above supremum by $\|\kappa\|_{\infty}$. 
Proof. Let $x \in \Omega$ and $y \in \Pi(x)$. Then the disk of centre $x$ and radius $d(x)$ is tangent to $\partial \Omega$ at $y$. Therefore, we have either $\kappa(y) \leq 0$ or $1 / \kappa(y) \geq d(x)$. So, $\kappa(y) d(x) \leq 1$.

If we assume, next, that $x \notin \bar{\Sigma}$, then $y$ belongs to the projection of $x+s \bar{D} d(x)$ for $s>0$ sufficiently small. Thus $\kappa(y) d(x+s D d(x)) \leq 1$. Since $d(x+s D d(x))=d(x)+s$ and $\kappa(x)=\kappa(y)$ by definition, we have $\kappa(x) d(x)<1$. For the last assertion see [21, Lemma 14.17].

We will need a more detailed description of the singular set $\Sigma$. Let us recall that $\Sigma=$ $\Sigma^{1} \cup \Sigma^{2}$, where $\Sigma^{i}(i=1,2)$ is the set of points $x \in \Sigma$ with magnitude $i$, that is, such that the dimension of $D^{+} d(x)$ is equal to $i$. Let us also define the set $\Gamma$ of (regular) conjugate points as

$$
\Gamma=\{x \in \Omega \backslash \Sigma: d(x) \kappa(x)=1\} .
$$

Notice that a point $x \in \Omega \backslash \Sigma$ belongs to $\Gamma$ if and only if

$$
\Pi(x)=\left\{x-\frac{1}{\kappa(x)} D d(x)\right\} .
$$

Proposition 2.8. Under assumption (6), we have $\bar{\Sigma} \subset \Omega$ and $\bar{\Sigma}=\Sigma \cup \Gamma$.

Proof. Let $x \in \Sigma$ and $y, z$ be two distinct elements of $\Pi(x)$. Then

$$
x=y+d(x) \operatorname{Dd}(y)=z+d(x) \operatorname{Dd}(z) .
$$

Therefore, recalling Remark 2.7

$$
|y-z|=d(x)|D d(y)-D d(z)| \leq d(x) K|y-z|
$$

for some constant $K>0$ independent of $x$. We have thus proved that $d(x) \geq 1 / K$ for every $x \in \Sigma$. So, $\bar{\Sigma} \subset \Omega$. Furthermore, the inclusion $\Gamma \subset \bar{\Sigma}$ is a straightforward consequence of the strict inequality in Proposition 2.6

In order to prove the inclusion $\bar{\Sigma} \subset \Sigma \cup \Gamma$, let $\left\{x_{n}\right\}$ be a sequence of singular points converging to a point $x \in \Omega \backslash \Sigma$. We claim that $d(x) \kappa(x)=1$. To see this, let $y_{n}$ and $z_{n}$ be two distinct points in $\Pi\left(x_{n}\right)$. Then both $\left\{y_{n}\right\}$ and $\left\{z_{n}\right\}$ must converge to $\Pi(x)$ as $n \rightarrow \infty$. Also, passing to a subsequence,

$$
\lim _{n \rightarrow \infty} \frac{y_{n}-z_{n}}{\left|y_{n}-z_{n}\right|}=\theta
$$

for some unit vector $\theta \in \mathbb{R}^{2}$. From identity 10 applied to $x_{n}, y_{n}$ and $z_{n}$, we have

$$
0=\frac{y_{n}-z_{n}}{\left|y_{n}-z_{n}\right|}+d\left(x_{n}\right) \frac{D d\left(y_{n}\right)-D d\left(z_{n}\right)}{\left|y_{n}-z_{n}\right|} .
$$

Hence, taking the limit as $n \rightarrow \infty$ we conclude that $0=\theta+d(x) D^{2} d(\Pi(x)) \theta$. Therefore, $-1 / d(x)$ is a nonzero eigenvalue of $D^{2} d(\Pi(x))$, a matrix of the form $-\kappa(x) q \otimes q$ by Proposition 2.6 So, $-1 / d(x)=-\kappa(x)$, as claimed.

The following result ensures that segments of minimal length joining a point to $\partial \Omega$ contain no singular or conjugate points in their interior. 
Proposition 2.9. Let $x \in \Omega$ and $y \in \Pi(x)$. Then $\bar{\Sigma} \cap] y, x[=\emptyset$.

Proof. We already know that $\Sigma \cap] y, x[=\emptyset$ by Proposition $2.2(\mathrm{~b})$, and that $\kappa(y) d(x) \leq 1$ by Proposition 2.6. Since $\kappa(y) d(z)<1$ for every $z \in] y, x[$, we conclude that $z \notin \Gamma$.

The following proposition, which will be crucial to our analysis, is an adaptation of some of the results of [1] describing propagation of singularities for semiconcave functions.

Proposition 2.10. Let $x_{0} \in \Sigma$, and let $p_{0}, q_{0}$ be two distinct limiting gradients at $x_{0}$ such that the segment $\left[p_{0}, q_{0}\right]$ is a face of $D^{+} d\left(x_{0}\right)$. Let $n_{0}$ be a nonzero vector satisfying

$$
\left\langle p, n_{0}\right\rangle \leq\left\langle p_{0}, n_{0}\right\rangle=\left\langle q_{0}, n_{0}\right\rangle \quad \forall p \in D^{+} d\left(x_{0}\right) .
$$

Then there exist a number $\eta>0$ and a Lipschitz arc $\boldsymbol{x}:[0, \eta] \rightarrow \Omega$ such that

$$
\boldsymbol{x}(0)=x_{0}, \quad \dot{\boldsymbol{x}}(0)=-n_{0}, \quad \boldsymbol{x}(s) \in \Sigma \quad \forall s \in[0, \eta] .
$$

Moreover, $\boldsymbol{x}\left(s_{n}\right) \in \Sigma^{1}$ for some sequence $s_{n} \downarrow 0$, and

$$
D^{+} d\left(\boldsymbol{x}\left(s_{n}\right)\right)=\left[p_{n}, q_{n}\right] \quad \forall n \geq 0,
$$

where $p_{n} \rightarrow p_{0}$ and $q_{n} \rightarrow q_{0}$ as $n \rightarrow \infty$.

Proof. The existence of a singular arc $x$ satisfying (11) follows from Lemma 4.5 and Theorem 4.2 of [1], where a bound of the form diam $D^{+} d(x(s)) \geq \delta$ is also deduced for some $\delta>0$ and every $s \in[0, \eta]$.

To prove the last part of the conclusion, we note that, for any $\epsilon>0, \mathcal{H}^{1}(\boldsymbol{x}([0, \epsilon]))>0$ because $\dot{\boldsymbol{x}}(0) \neq 0$ and $\boldsymbol{x}$ is Lipschitz continuous. Since $\Sigma^{2}$ is at most countable (see, e.g., [14]), we conclude that $\mathcal{H}^{1}\left(\boldsymbol{x}([0, \epsilon]) \cap \Sigma^{1}\right)>0$ for any $\epsilon>0$. Consequently, there exists a sequence $s_{n} \downarrow 0$ such that $\boldsymbol{x}\left(s_{n}\right) \in \Sigma^{1}$ for every $n \in \mathbb{N}$. Set $D^{+} d\left(\boldsymbol{x}\left(s_{n}\right)\right)=\left[p_{n}, q_{n}\right]$, choosing $p_{n}$ so that $\left\langle p_{n}, p_{0}\right\rangle \geq\left\langle q_{n}, p_{0}\right\rangle$. Notice that, in particular, $\left|p_{n}-q_{n}\right| \geq \delta$. Now, consider converging subsequences of $\left\{p_{n}\right\}$ and $\left\{q_{n}\right\}$ (labelled like the original sequences) and denote by $p^{*}$ and $q^{*}$ their respective limits. Applying [3, Theorem 2.1], we deduce that

$$
p^{*}, q^{*} \in \arg \max _{p \in D^{+} d\left(x_{0}\right)}\left\langle p, n_{0}\right\rangle=\left[p_{0}, q_{0}\right] .
$$

Since $p^{*}$ and $q^{*}$ belong to $D^{*} d\left(x_{0}\right)$, we conclude that $p^{*}, q^{*} \in\left\{p_{0}, q_{0}\right\}$. Moreover, $\left|p^{*}-q^{*}\right| \geq \delta$ and $\left\langle p^{*}, p_{0}\right\rangle \geq\left\langle q^{*}, p_{0}\right\rangle$. This forces $(p, q)=\left(p_{0}, q_{0}\right)$.

Remark 2.11. Elementary geometric arguments show that $D^{+} d\left(x_{0}\right)$ has a 1-dimensional exposed face-and so, owing to Proposition 2.10 $x_{0}$ is the inital point of a nonconstant Lipschitz singular arc-if and only if $D^{+} d\left(x_{0}\right)$ fails to cover the closed unit ball $\bar{B}_{1}$. On the other hand, in view of $[5), D^{+} d\left(x_{0}\right)=\bar{B}_{1}$ if and only if $\partial D^{+} d\left(x_{0}\right)=D^{*} d\left(x_{0}\right)$. By [1. Theorem 6.2], the last identity is necessary and sufficient for $\Sigma$ to be a singleton or, equivalently, for $\Omega$ to coincide with $B_{R}\left(x_{0}\right)$, where $R=d\left(x_{0}\right)$. In fact, the equivalence between $\Sigma$ being a singleton and the identity $\Omega=B_{R}\left(x_{0}\right)$ follows from a classical result of Motzkin's [26].

In conclusion, either $\Omega=B_{R}\left(x_{0}\right)$ or every singularity propagates along Lipschitz arcs. Moreover, by the last part of Proposition 2.10, $\Sigma^{1}$ is dense in $\Sigma$ in the latter case. 
We conclude this section with a regularity result for the normal distance $\tau$.

Theorem 2.12. Let $\Omega$ be a bounded domain in $\mathbb{R}^{2}$ with boundary of class $\mathcal{C}^{2,1}$. Then the map $\tau$ defined in (7) is Lipschitz continuous on $\partial \Omega$.

The first author became aware of the above property from [25]. A proof of this result for $\mathcal{C}^{\infty}$ smooth submanifolds of an $n$-dimensional smooth manifold is given in [23]. In the Appendix, we provide an independent proof of Theorem 2.12 based on Proposition 2.10

Hereafter, we will denote by $\operatorname{Lip}(\tau)$ the Lipschitz seminorm of $\tau$ on $\partial \Omega$. Since $x \mapsto$ $x+\tau(x) D d(x)$ maps $\partial \Omega$ onto $\bar{\Sigma}$, a straightforward application of Theorem 2.12 is that the 1-dimensional Hausdorff measure of $\bar{\Sigma}$ is finite (see also [25]):

Corollary 2.13. Let $\Omega$ be a bounded domain in $\mathbb{R}^{2}$ with boundary of class $\mathcal{C}^{2,1}$. Then

$$
\mathcal{H}^{1}(\bar{\Sigma}) \leq k_{\Omega} \mathcal{H}^{1}(\partial \Omega)<\infty
$$

where $k_{\Omega} \geq 0$ is a constant depending on $\operatorname{Lip}(\tau)$ and on the quantity $\|\kappa\|_{\infty}$ defined in Remark 2.7

For less regular domains the Lipschitz continuity of $\tau$ may fail, but continuity is preserved as we show below.

Lemma 2.14. Assume (6). Then the map $\tau$, extended to 0 on $\bar{\Sigma}$, is continuous in $\bar{\Omega}$.

Proof. We only need to show that $\tau$ is upper semicontinuous in $\bar{\Omega}$, lower semicontinuity being a direct consequence of $\bar{\Sigma}$ being closed. For this purpose, consider a sequence $\left\{x_{n}\right\}$ in $\bar{\Omega} \backslash \bar{\Sigma}$, converging to some point $x \in \bar{\Omega}$, and suppose by contradiction $t^{*}:=$ $\lim _{n} \tau\left(x_{n}\right)>\tau(x)$. In particular, this implies that $t^{*}$ is positive. We can also assume, without loss of generality, that $\left\{D d\left(x_{n}\right)\right\}$ converges, say to $p$. Let $\left.\bar{t} \in\right] \tau(x), t^{*}[$. Then $d$ is differentiable at $x_{n}+\bar{t} D d\left(x_{n}\right)$ by definition. Thus, for $n$ large enough,

$$
\Pi\left(x_{n}+\bar{t} D d\left(x_{n}\right)\right)=\Pi\left(x_{n}\right)=\left\{x_{n}-d\left(x_{n}\right) D d\left(x_{n}\right)\right\} .
$$

Taking the limit as $n \rightarrow \infty$, we obtain $x-d(x) p \in \Pi(x+\bar{t} p)$. So, $x+\tau(x) p$ belongs to the interior of the segment $[x-d(x) p, x+\bar{t} p]$. Since $x+\tau(x) p \in \bar{\Sigma}$, this contradicts Proposition 2.9

We conclude this section with an approximation result. Roughly speaking, we need to make sure that both the singular set and the normal distance are stable for convergence in the $\mathcal{C}^{2}$ topology. We begin by defining the signed distance from $\partial \Omega$ as

$$
\boldsymbol{d}_{\Omega}(x)= \begin{cases}d_{\partial \Omega}(x) & \text { if } x \in \bar{\Omega}, \\ -d_{\partial \Omega}(x) & \text { if } x \in \mathbb{R}^{2} \backslash \bar{\Omega} .\end{cases}
$$

We say that a sequence $\left\{\Omega_{n}\right\}$ of sets satisfying $(6)$ converges to $\Omega$ in the $\mathcal{C}^{2}$ topology if the boundary of $\Omega_{n}$ converges to the boundary of $\Omega$ for the Hausdorff distance and if $\boldsymbol{d}_{\Omega_{n}}, D \boldsymbol{d}_{\Omega_{n}}$ and $D^{2} \boldsymbol{d}_{\Omega_{n}}$ converge to $\boldsymbol{d}_{\Omega}, D \boldsymbol{d}_{\Omega}$ and $D^{2} \boldsymbol{d}_{\Omega}$, uniformly in a neighbourhood of $\partial \Omega$. 
Proposition 2.15. Let $\left\{\Omega_{n}\right\}$ be a sequence of sets satisfying (6). For any $n \in \mathbb{N}$, denote by $\Sigma_{n}$ and $\tau_{n}$, respectively, the singular set of $\Omega_{n}$ and the normal distance to $\bar{\Sigma}_{n}$. If $\left\{\Omega_{n}\right\}$ converges to $\Omega$ in the $\mathcal{C}^{2}$ topology, then $\left\{\bar{\Sigma}_{n}\right\}$ converges to $\bar{\Sigma}$ in the Hausdorff topology, and $\left\{\tau_{n}\right\}$ converges to $\tau$ uniformly on all compact subsets of $\Omega$.

Proof. Let us prove, first, that the upper limit of $\left\{\bar{\Sigma}_{n}\right\}$ is contained in $\bar{\Sigma}$. For this it suffices to show that if a sequence $\left\{x_{n}\right\}$ in $\Sigma_{n}$ converges to a point $x \in \bar{\Omega}$, then $x$ belongs to $\bar{\Sigma}$. Indeed, let $y_{n}$ and $z_{n}$ be two distinct projections of $x_{n}$ onto $\partial \Omega_{n}$. Without loss of generality we can assume that both $\left\{y_{n}\right\}$ and $\left\{z_{n}\right\}$ converge to points of $\Pi(x)$, say $y$ and $z$ respectively. If $y \neq z$, then $x$ belongs to $\Sigma$ and our claim follows. So, suppose $x \in \bar{\Omega} \backslash \Sigma$ and $y=z$. Since $y_{n}+\boldsymbol{d}_{\Omega_{n}}\left(x_{n}\right) D \boldsymbol{d}_{\Omega_{n}}\left(y_{n}\right)=z_{n}+\boldsymbol{d}_{\Omega_{n}}\left(x_{n}\right) D \boldsymbol{d}_{\Omega_{n}}\left(z_{n}\right)$, we have

$$
\frac{y_{n}-z_{n}}{\left|y_{n}-z_{n}\right|}=-\boldsymbol{d}_{\Omega_{n}}\left(x_{n}\right) \frac{D \boldsymbol{d}_{\Omega_{n}}\left(y_{n}\right)-D \boldsymbol{d}_{\Omega_{n}}\left(z_{n}\right)}{\left|y_{n}-z_{n}\right|} .
$$

The sequence on the left-hand side above will converge, up to replacement with a subsequence, to some unit vector $\theta \in \mathbb{R}^{2}$. Thus, passing to the limit in 13 we obtain $\theta=-\boldsymbol{d}_{\Omega}(x) D^{2} \boldsymbol{d}_{\Omega}(y) \theta$. Hence, recalling the structure of the hessian matrix $D^{2} d(y)$, we conclude that $d(x) \kappa(x)=d(x) \kappa(y)=1$. Therefore, $x$ belongs to $\bar{\Sigma}$.

Now, let us prove that the lower limit of the sequence $\left\{\bar{\Sigma}_{n}\right\}$ contains $\bar{\Sigma}$. For this, it suffices to show that $\Sigma \subset \liminf \Sigma_{n}$. Let $x \in \Omega \backslash \liminf \Sigma_{n}$. Then there exists a subsequence $\left\{\Sigma_{n_{k}}\right\}$ such that, for some $\varepsilon>0, B_{\varepsilon}(x) \subset \Omega \backslash \Sigma_{n_{k}}$. We claim that $B_{\varepsilon / 2}(x)$ $\cap \Sigma=\emptyset$. For let $z \in B_{\varepsilon / 2}(x)$ and set $y_{k}=\Pi_{\partial \Omega_{n_{k}}}(z)$. Since

$$
z+\varepsilon \frac{z-y_{k}}{2 d_{\Omega_{n_{k}}}(z)} \in B_{\varepsilon / 2}(z) \subset B_{\varepsilon}(x) \subset \Omega \backslash \Sigma_{n_{k}},
$$

$y_{k}$ is also the unique projection of $z+\varepsilon\left(z-y_{k}\right) / 2 \boldsymbol{d}_{\Omega_{n_{k}}}(z)$ onto $\partial \Omega_{n_{k}}$. Now, a subsequence of $\left\{y_{k}\right\}$ will converge to some point $y \in \partial \Omega$ belonging to both projections $\Pi(z)$ and $\Pi\left(z+\varepsilon(z-y) / 2 \boldsymbol{d}_{\Omega}(z)\right)$. Therefore, $z \notin \bar{\Sigma}$ owing to Proposition 2.9, and our claim is proved together with the convergence of $\bar{\Sigma}_{n}$ to $\bar{\Sigma}$.

We omit the proof that $\left\{\tau_{n}\right\}$ converges to $\tau$, because the reasoning has much in common with the proof of Lemma2.14.

\section{Existence}

In this section we shall prove that the pair $\left(d, v^{f}\right)$, where

$$
v^{f}(x)=\int_{0}^{\tau(x)} f(x+t D d(x)) \frac{1-(d(x)+t) \kappa(x)}{1-d(x) \kappa(x)} d t \quad \forall x \in \Omega \backslash \bar{\Sigma}
$$

and $v^{f} \equiv 0$ on $\bar{\Sigma}$, is a solution of system 22. Before getting down to rigorous arguments, a formal derivation of such a formula might be in order. Suppose $(d, v)$ is a smooth 
solution of 22, with $v$ vanishing on $\bar{\Sigma}$. Let us compute, for a given point $x \in \Omega \backslash \bar{\Sigma}$ and for any $t \in(0, \tau(x))$, the derivative

$$
\begin{aligned}
\frac{d}{d t} v(x+t D d(x)) & =\langle D v(x+t D d(x)), D d(x)\rangle \\
& =-v(x+t D d(x)) \Delta d(x+t D d(x))-f(x+t D d(x))
\end{aligned}
$$

$($ recall that $D d(x+t D d(x))=D d(x))$. Now, observe that

$$
\Delta d(x+t D d(x))=-\frac{\kappa(x)}{1-(d(x)+t) \kappa(x)}
$$

since $\kappa(x+t D d(x))=\kappa(x)$ and $d(x+t D d(x))=d(x)+t$. Hence, the function $V(t):=v(x+t D d(x))$ satisfies the Cauchy problem

$$
\left\{\begin{array}{l}
V^{\prime}(t)-\frac{\kappa(x)}{1-(d(x)+t) \kappa(x)} V(t)+f(x+t D d(x))=0, \\
V(\tau(x))=0 .
\end{array}\right.
$$

Thus, solving the above problem and noting that $v(x)=V(0)$, we conclude that $v$ must be given by formula (14).

We begin the actual proof with two preliminary results, the first describing continuity and differentiability properties of $v^{f}$, and the other providing an approximation result for the characteristic function of a compact set, in the spirit of capacity theory.

Proposition 3.1. Let $\Omega \subset \mathbb{R}^{2}$ be a bounded domain with boundary of class $\mathcal{C}^{2}$ and $f \geq 0$ be a continuous function in $\Omega$. Then $v^{f}$ is a locally bounded continuous function in $\Omega$. Moreover, in any set $\Omega_{\varepsilon}:=\{x \in \Omega: d(x)>\varepsilon\}, \varepsilon>0, v^{f}$ satisfies the bound

$$
0 \leq v^{f}(x) \leq\|f\|_{\infty, \Omega_{\varepsilon}}\left[1+\left\|[\kappa]_{-}\right\|_{\mathcal{C}(\partial \Omega)} \operatorname{diam} \Omega\right] \tau(x) \quad \forall x \in \Omega_{\varepsilon},
$$

where $\left\|[\kappa]_{-}\right\|_{\mathcal{C}(\partial \Omega)}:=\max _{x \in \partial \Omega}[\kappa(x)]_{-}$.

If, in addition, $\partial \Omega$ is of class $\mathcal{C}^{2,1}$ and $f$ is Lipschitz continuous in $\Omega$, then $v^{f}$ is locally Lipschitz continuous in $\Omega \backslash \bar{\Sigma}$ and satisfies

$$
-\operatorname{div}\left(v^{f}(x) D d(x)\right)=f(x)
$$

at each point $x \in \Omega \backslash \bar{\Sigma}$ at which $v^{f}$ is differentiable.

Remark 3.2. Since $d$ is $\mathcal{C}^{2}$ in $\Omega \backslash \bar{\Sigma}$, equality 16 reads

$$
\left\langle D v^{f}(x), D d(x)\right\rangle+v^{f}(x) \Delta d(x)+f(x)=0 .
$$

Moreover, a straightforward consequence of Proposition 3.1 is that $-\operatorname{div}\left(v^{f} D d\right)=f$ in the sense of distributions in $\Omega \backslash \bar{\Sigma}$ as soon as $f$ is Lipschitz and $\partial \Omega$ of class $\mathcal{C}^{2,1}$. 
Proof. We note, first, that the maps $D d, \tau$ and $\kappa$ are continuous in $\Omega \backslash \bar{\Sigma}$ since $\Omega$ has a $\mathcal{C}^{2}$ boundary. Hence, when $f$ is continuous, so is $v^{f}$ in $\Omega \backslash \bar{\Sigma}$.

Let us now prove that $v^{f}$ is continuous on $\bar{\Sigma}$. Observe that, for any $x \notin \bar{\Sigma}$, the term

$$
\frac{1-(d(x)+t) \kappa(x)}{1-d(x) \kappa(x)}=\frac{1-d(x+t D d(x)) \kappa(x)}{1-d(x) \kappa(x)}, \quad 0<t<\tau(x),
$$

is nonnegative by Proposition 2.6 A simple computation shows that it is also bounded by $1+\left\|[\kappa]_{-}\right\|_{\mathcal{C}(\partial \Omega)} \tau(x)$. Indeed, either $\kappa(x) \geq 0$ and so the above fraction is less than 1 , or $\kappa(x)<0$ and then

$$
\frac{1-(d(x)+t) \kappa(x)}{1-d(x) \kappa(x)}=1-\frac{t \kappa(x)}{1-d(x) \kappa(x)} \leq 1+\left\|[\kappa]_{-}\right\|_{\mathcal{C}(\partial \Omega)} \tau(x) .
$$

This proves (15) if we recall that $x+t D d(x) \in \Omega_{\varepsilon}$ whenever $x \in \Omega_{\varepsilon}$ and $0 \leq t \leq \tau(x)$. The continuity of $v^{f}$ on $\bar{\Sigma}$ is an immediate consequence of 15 .

Next, let $\partial \Omega$ be of class $\mathcal{C}^{2,1}$ and $f$ be Lipschitz. Then Theorem 2.12 ensures that $\tau$ is Lipschitz on $\partial \Omega$. Therefore, $\tau=\tau \circ \Pi-d$ is locally Lipschitz in $\bar{\Omega} \backslash \bar{\Sigma}$, and so also is $v^{f}$.

Finally, let us check the validity of 16 at every differentiability point $x$ of $v^{f}$ in the open set $\Omega \backslash \bar{\Sigma}$. We note that, at any such point $x$,

$$
\left\langle D v^{f}(x), D d(x)\right\rangle=\left.\frac{d}{d \lambda} v^{f}(x+\lambda D d(x))\right|_{\lambda=0} .
$$

But $\tau(x+\lambda D d(x))=\tau(x)-\lambda$ and $d(x+\lambda D d(x))=d(x)+\lambda$ for $\lambda>0$ sufficiently small. So,

$$
\begin{aligned}
v^{f}(x+\lambda D d(x)) & =\int_{0}^{\tau(x)-\lambda} f(x+(t+\lambda) D d(x)) \frac{1-(d(x)+\lambda+t) \kappa(x)}{1-(d(x)+\lambda) \kappa(x)} d t \\
& =\int_{\lambda}^{\tau(x)} f(x+t D d(x)) \frac{1-(d(x)+t) \kappa(x)}{1-(d(x)+\lambda) \kappa(x)} d t .
\end{aligned}
$$

Therefore,

$$
\begin{aligned}
\left\langle D v^{f}(x), D d(x)\right\rangle & =-f(x)+\int_{0}^{\tau(x)} f(x+t D d(x)) \frac{1-(d(x)+t) \kappa(x)}{(1-d(x) \kappa(x))^{2}} \kappa(x) d t \\
& =-f(x)-v^{f}(x) \Delta d(x),
\end{aligned}
$$

where we have taken into account the identity

$$
\Delta d(x)=-\frac{\kappa(x)}{1-d(x) \kappa(x)} \quad \forall x \in \Omega \backslash \bar{\Sigma},
$$

which follows from Proposition 2.6 We have thus obtained (17) —an equivalent version of $(16)$ - and completed the proof. 
Proposition 3.3. Let $K$ be a compact subset of $\mathbb{R}^{2}$ such that $\mathcal{H}^{1}(K)<\infty$. Then there exists a sequence $\left\{\xi_{k}\right\}$ of functions in $W^{1,1}\left(\mathbb{R}^{2}\right)$ with compact support such that

(a) $0 \leq \xi_{k} \leq 1$ for every $k \in \mathbb{N}$;

(b) $\operatorname{dist}\left(\operatorname{spt}\left(\xi_{k}\right), K\right) \rightarrow 0$ as $k \rightarrow \infty$;

(c) $K \subset \operatorname{int}\left\{x \in \mathbb{R}^{2}: \xi_{k}(x) \geq 1\right\}$ for every $k \in \mathbb{N}$;

(d) $\xi_{k} \rightarrow 0$ in $L^{1}\left(\mathbb{R}^{2}\right)$ as $k \rightarrow \infty$;

(e) $\int_{\mathbb{R}^{2}}\left|D \xi_{k}\right| d x \leq \frac{3}{2} \pi\left(\mathcal{H}^{1}(K)+1 / k\right)$ for every $k \in \mathbb{N}$.

The standard notations dist, spt and int stand for distance (between two sets), support (of a function) and interior (of a set), respectively. We give a proof of the proposition for the reader's convenience.

Proof. Since $\mathcal{H}^{1}(K)<\infty$, for any fixed $k \in \mathbb{N}$ there exists a sequence $\left\{x_{i}^{(k)}\right\}_{i \in \mathbb{N}}$ of points in $K$ and a sequence $\left\{r_{i}^{(k)}\right\}_{i \in \mathbb{N}}$ of radii such that

- $0<r_{i}^{(k)} \leq 1 / k$ and $\sum_{i} r_{i}^{(k)} \leq \frac{1}{2}\left(\mathcal{H}^{1}(K)+1 / k\right)$;

- $K \subset \operatorname{int}\left(\bigcup_{i} B_{r_{i}^{(k)}}\left(x_{i}^{(k)}\right)\right)$.

Now, define, for any $x \in \mathbb{R}^{2}$,

$$
\xi_{i}^{(k)}(x)=\left[1-\frac{1}{r_{i}^{(k)}}\left(\left|x-x_{i}^{(k)}\right|-r_{i}^{(k)}\right)_{+}\right]_{+}, \quad \xi_{k}(x)=\sup _{i \in \mathbb{N}} \xi_{i}^{(k)}(x),
$$

and observe that

$$
\operatorname{spt}\left(\xi_{i}^{(k)}\right)=\bar{B}_{2 r_{i}^{(k)}}\left(x_{i}^{(k)}\right), \quad \operatorname{spt}\left(D \xi_{i}^{(k)}\right)=\bar{B}_{2 r_{i}^{(k)}}\left(x_{i}^{(k)}\right) \backslash B_{r_{i}^{(k)}}\left(x_{i}^{(k)}\right),
$$

Then $\xi_{k} \in L^{1}\left(\mathbb{R}^{2}\right)$ since $0 \leq \xi_{k} \leq 1$ and $\xi_{k}$ has compact support. Moreover, an easy computation shows that $\int_{\mathbb{R}^{2}}\left|D \xi_{i}^{(k)}\right| d x=3 \pi r_{i}^{(k)}$. So, applying [16, Lemma 2, p. 148], we have

$$
\int_{\mathbb{R}^{2}}\left|D \xi_{k}\right| d x \leq \int_{\mathbb{R}^{2}} \sup _{i}\left|D \xi_{i}^{(k)}\right| d x \leq \sum_{i} \int_{\mathbb{R}^{2}}\left|D \xi_{i}^{(k)}\right| d x \leq \frac{3}{2} \pi\left(\mathcal{H}^{1}(K)+\frac{1}{k}\right) .
$$

Therefore, $\xi_{k} \in W^{1,1}\left(\mathbb{R}^{2}\right)$ and (e) holds true. Properties (b) and (c) are true by construction. Finally, (d) follows by Lebesgue's Theorem because $0 \leq \xi_{k} \leq 1$ and $\xi_{k}(x)=0$ for any point $x \notin K$ and $k$ large enough.

Proof of Theorem 1.1 (existence). We will prove that the pair $\left(d, v^{f}\right)$, with $v^{f}$ defined by [14), is a solution of system [2]. Let us point out, to begin with, that $d$ is a viscosity solution of the eikonal equation in $\Omega$, and so, a fortiori, in the open set $\left\{x \in \Omega: v^{f}(x)\right.$ $>0\}$. Therefore, what actually remains to be shown is that

$$
\int_{\Omega} f \phi d x=\int_{\Omega} v^{f}\langle D d, D \phi\rangle d x \quad \forall \phi \in \mathcal{C}_{\mathrm{c}}^{\infty}(\Omega) .
$$


Assume, first, that $f$ is Lipschitz and $\Omega$ has a $\mathcal{C}^{2,1}$ boundary. Since $\mathcal{H}^{1}(\bar{\Sigma})<\infty$ by Proposition 2.13, we can apply Proposition 3.3 with $K=\bar{\Sigma}$ to construct a sequence $\left\{\xi_{k}\right\}$ enjoying properties (a)-(d). Let $\phi \in \mathcal{C}_{\mathrm{c}}^{\infty}(\Omega)$ be a test function, and set $\phi_{k}=\phi\left(1-\xi_{k}\right)$. Notice that, for $k$ large enough, $\operatorname{spt}\left(\phi_{k}\right) \subset \subset \Omega \backslash \bar{\Sigma}$. This follows from (a), (b) and from the fact that $\bar{\Sigma} \subset \Omega$ (see Proposition 2.8). Then Proposition 3.1 and Rademacher's Theorem imply that $-\operatorname{div}\left(v^{f} D d\right)=f$ a.e. in $\Omega \backslash \bar{\Sigma}$. So, multiplying this equation by $\phi_{k}$ and integrating by parts, we obtain

$$
\int_{\Omega} f \phi_{k} d x=\int_{\Omega} v^{f}\left(1-\xi_{k}\right)\langle D d, D \phi\rangle d x-\int_{\Omega} v^{f} \phi\left\langle D d, D \xi_{k}\right\rangle d x
$$

We claim that the rightmost term above goes to 0 as $k \rightarrow \infty$. Indeed,

$$
\begin{aligned}
\left|\int_{\Omega} v^{f} \phi\left\langle D d, D \xi_{k}\right\rangle d x\right| & \leq\|\phi\|_{\infty, \Omega}\left\|v^{f}\right\|_{\infty, \operatorname{spt}\left(\xi_{k}\right)} \int_{\Omega}\left|D \xi_{k}\right| d x \\
& \leq C\|\phi\|_{\infty, \Omega}\left\|v^{f}\right\|_{\infty, \operatorname{spt}\left(\xi_{k}\right)}
\end{aligned}
$$

where $C=\frac{3}{2} \pi\left(\mathcal{H}^{1}(K)+1 / k\right)$. Now, using property (a) of the proposition and the fact that $v^{f}$ is a continuous function vanishing on $\bar{\Sigma}$, we conclude that $\left\|v^{f}\right\|_{\infty, \operatorname{spt}\left(\xi_{k}\right)} \rightarrow 0$ as $k \rightarrow \infty$. This proves our claim. The conclusion (18) immediately follows since, by (a) and (c),

$$
\int_{\Omega} f \phi_{k} d x \rightarrow \int_{\Omega} f \phi d x \text { and } \int_{\Omega} v^{f}\left(1-\xi_{k}\right)\langle D d, D \phi\rangle d x \rightarrow \int_{\Omega} v^{f}\langle D d, D \phi\rangle d x
$$

as $k \rightarrow \infty$.

Finally, the extra assumptions that $\partial \Omega$ be of class $\mathcal{C}^{2,1}$ and $f$ be Lipschitz in $\Omega$ can be easily removed by an approximation argument based on the lemma below.

Let $\left\{\Omega_{n}\right\}$ be a sequence of open domains, with $\mathcal{C}^{2,1}$ boundary, converging to $\Omega$ in the $\mathcal{C}^{2}$ topology, and let $\left\{f_{n}\right\}$ be a sequence of Lipschitz functions in $\Omega_{n}$ converging to $f$, uniformly on all compact subsets of $\Omega$. Denote by $\Sigma_{n}$ and $\tau_{n}$, respectively, the singular set of $\Omega_{n}$ and the normal distance to $\bar{\Sigma}_{n}$. Define $v_{n}(x)=0$ for every $x \in \bar{\Sigma}_{n}$ and

$$
v_{n}(x)=\int_{0}^{\tau_{n}(x)} f_{n}\left(x+t D \boldsymbol{d}_{\Omega_{n}}(x)\right) \frac{1-\left(\boldsymbol{d}_{\Omega_{n}}(x)+t\right) \kappa_{n}(x)}{1-\boldsymbol{d}_{\Omega_{n}}(x) \kappa_{n}(x)} d t \quad \forall x \in \Omega_{n} \backslash \bar{\Sigma}_{n},
$$

where $\kappa_{n}(x)$ stands for the curvature of $\partial \Omega_{n}$ at the projection of $x$.

Lemma 3.4. $\left\{v_{n}\right\}$ converges to $v^{f}$ in $L_{\mathrm{loc}}^{1}(\Omega)$.

Proof. Since, owing to $(15)$, the sequence $\left\{v_{n}\right\}$ is locally uniformly bounded in $\Omega$, it suffices to prove that it converges uniformly to $v$ on every compact subset of $\Omega$. For this, recall that, on account of Proposition 2.15. $\left\{\bar{\Sigma}_{n}\right\}$ converges to $\bar{\Sigma}$ in the Hausdorff topology and $\left\{\tau_{n}\right\}$ converges to $\tau$ uniformly on all compact subsets of $\Omega$. Then our assumptions imply that $\left\{\kappa_{n}\right\}$ converges to $\kappa$ uniformly on every compact subset of $\Omega \backslash \bar{\Sigma}$, and so does $\left\{v_{n}\right\}$ to $v^{f}$. To complete the proof it suffices to combine the above local uniform convergence in $\Omega \backslash \bar{\Sigma}$ with the estimate

$$
0 \leq v_{n}(x) \leq\left\|f_{n}\right\|_{\infty, \Omega_{\varepsilon}}\left(1+\left\|\left[\kappa_{n}\right]_{-}\right\|_{\mathcal{C}\left(\partial \Omega_{n}\right)} \operatorname{diam} \Omega_{n}\right) \tau_{n}(x) \quad \forall x \in \Omega_{\varepsilon},
$$

which allows estimating $v_{n}$ on any neighbourhood of $\bar{\Sigma}$. 


\section{Uniqueness}

In this section we will prove that if $(u, v)$ is a solution of system 22, then $v \equiv v^{f}$ (given by (47) and $u \equiv d$ in $\Omega_{v}:=\{x \in \Omega: v(x)>0\}$. We begin by showing the last statement.

Proposition 4.1. If $(u, v)$ is a solution of system (2), then $u \equiv d$ in $\Omega_{v}$.

Proof. Since $\|D u\|_{\infty, \Omega} \leq 1$ and $u=0$ on $\partial \Omega$, we have $u \leq d$ in $\Omega$ because, in view of Remark 2.4 $d$ is the largest function with such properties. Moreover, since $u$ solves the eikonal equation in $\Omega_{v}$, Proposition 2.2 ensures that

$$
u(x)=\min _{\left.y \in \partial \Omega_{v},\right] x, y\left[\subset \Omega_{v}\right.}\{u(y)+|y-x|\} \quad \forall x \in \Omega_{v} .
$$

We will argue by contradiction, supposing $u\left(x_{0}\right)<d\left(x_{0}\right)$ for some point $x_{0} \in \Omega_{v}$. Without loss of generality, $x_{0}$ may be assumed to be a point of differentiability of both $u$ and $D u$ (recall Remark 2.3. Let $y_{0} \in \partial \Omega_{v}$ be such that

$$
] x_{0}, y_{0}\left[\subset \Omega_{v}, \quad u\left(x_{0}\right)=u\left(y_{0}\right)+\left|y_{0}-x_{0}\right| .\right.
$$

Notice that $y_{0} \notin \partial \Omega$ because, otherwise, one would have $u\left(y_{0}\right)=0$, and so $u\left(x_{0}\right)=$ $\left|y_{0}-x_{0}\right|$, contrary to $u\left(x_{0}\right)<d\left(x_{0}\right)$.

Next, set $L:=\left\|D^{2} u\left(x_{0}\right)\right\|$ and fix $\varepsilon>0$ such that

$$
0<\varepsilon<\min \left\{1, \frac{v\left(x_{0}\right)}{16[1+(1+L) \operatorname{diam} \Omega]}\right\} .
$$

We claim that there exists $\rho>0$ such that the balls $B_{\rho}\left(x_{0}\right)$ and $B_{\rho}\left(y_{0}\right)$ are both contained in $\Omega$, and

$$
\begin{array}{ll}
\left|p-D u\left(x_{0}\right)\right| \leq 1 / 2 & \forall p \in D^{+} u(x), \forall x \in B_{\rho}\left(x_{0}\right), \\
v(x) \geq v\left(x_{0}\right) / 2 & \forall x \in B_{\rho}\left(x_{0}\right), \\
v(y) \leq \varepsilon & \forall y \in B_{\rho}\left(y_{0}\right) .
\end{array}
$$

Indeed, 21) follows from the upper semicontinuity of $D^{+} u$ (see Remark 2.3, while 22, and (23) can be obtained by a simple continuity argument since $v\left(x_{0}\right)>0$ and $v\left(y_{0}\right)=0$.

For brevity, set $e_{2}=D u\left(x_{0}\right)$ and let $e_{1} \in \mathbb{R}^{2}$ be such that $\left\{e_{1}, e_{2}\right\}$ is a positively oriented orthonormal basis of $\mathbb{R}^{2}$. Our choice of $x_{0}$ entails that for every sufficiently small $r>0$ there exists a point $x_{r} \in B_{r}\left(x_{0}\right)$ of differentiability of $u$ such that

$$
\begin{aligned}
\text { (i) } & \left|D u\left(x_{r}\right)-D u\left(x_{0}\right)-D^{2} u\left(x_{0}\right)\left(x_{r}-x_{0}\right)\right| \leq \varepsilon r, \\
\text { (ii) } & \left\langle e_{2}, x_{r}-x_{0}\right\rangle<0 \\
\text { (iii) } & \left\langle e_{1}, x_{r}-x_{0}\right\rangle>r / 2 .
\end{aligned}
$$

Now, fix $\left.y_{1} \in\right] x_{0}, y_{0}\left[\cap B_{\rho / 2}\left(y_{0}\right)\right.$, and let $r>0$ be so small that

$$
y_{r}:=x_{r}-\left|x_{0}-y_{1}\right| D u\left(x_{r}\right) \in B_{\rho}\left(y_{0}\right), \quad \operatorname{co}\left\{x_{0}, x_{r}, y_{1}, y_{r}\right\} \subset \Omega_{v} .
$$


Such an $r$ exists because $\left[x_{0}, y_{1}\right] \subset \Omega_{v}$ and

$$
\lim _{r \downarrow 0} y_{r}=x_{0}-\left|x_{0}-y_{1}\right| D u\left(x_{0}\right)=y_{1},
$$

since $x_{r} \rightarrow x_{0}$ and $D u\left(x_{r}\right) \rightarrow D u\left(x_{0}\right)$ as $r \downarrow 0$.

Finally, set $x_{1}=\Pi_{\left[x_{0}, y_{1}\right]}\left(x_{r}\right)$ and $\mathcal{C}=\operatorname{co}\left\{x_{1}, y_{1}, x_{r}, y_{r}\right\}$. We point out that, because of [24)(ii), $x_{1}$ belongs to the open segment ] $x_{0}, y_{1}$ [. The convex set $\mathcal{C}$ is a quadrilateral with sides $\left[x_{1}, x_{r}\right],\left[x_{r}, y_{r}\right],\left[y_{r}, y_{1}\right]$ and $\left[y_{1}, x_{1}\right]$. Moreover, $u$ is differentiable at any point $x \in\left[y_{1}, x_{1}\right]$ and $D u(x)=D u\left(x_{0}\right)$, as guaranteed by Proposition 2.2. b). Similarly, combining properties (c) and (b) of the same proposition shows that $u$ is differentiable at any point $x \in\left[x_{r}, y_{r}\right]$ and $D u(x)=D u\left(x_{r}\right)$.

Our next step would be to integrate the equation $-\operatorname{div}(v D u)=f$ over $\mathcal{C}$ and apply the Divergence Theorem. This reasoning needs the following approximation argument to be made rigorous. For any $\sigma>0$, consider the test function

$$
\psi_{\sigma}(x):=\left[1-\frac{1}{\sigma} d_{\mathcal{C}}(x)\right]_{+}, \quad x \in \mathbb{R}^{2},
$$

an element of $W^{1, \infty}\left(\mathbb{R}^{2}\right)$ with support $\mathcal{C}_{\sigma}:=\left\{x \in \mathbb{R}^{2}: d_{\mathcal{C}}(x) \leq \sigma\right\}$. Observe that, for $\sigma$ sufficiently small, $\psi_{\sigma} \in W_{\mathrm{c}}^{1, \infty}(\Omega)$. Also, $\operatorname{spt}\left(D \psi_{\sigma}\right)=\overline{\mathcal{C}_{\sigma} \backslash \mathcal{C}}$. Thus,

$$
\int_{\Omega} f \psi_{\sigma} d x=\int_{\Omega} v\left\langle D u, D \psi_{\sigma}\right\rangle d x=\int_{\mathcal{C}_{\sigma} \backslash \mathcal{C}} v\left\langle D u, D \psi_{\sigma}\right\rangle d x
$$

On the right-hand side of the above equality, we split the integration domain as $\mathcal{C}_{\sigma} \backslash \mathcal{C}=$ $E_{1}(\sigma) \cup E_{2}(\sigma) \cup E_{3}(\sigma) \cup E_{4}(\sigma)$, where

$$
\begin{aligned}
& E_{1}(\sigma)=\left\{x \in E: \Pi_{\mathcal{C}}(x) \in\right] x_{1}, y_{1}[\}, \\
& E_{2}(\sigma)=\left\{x \in E: \Pi_{\mathcal{C}}(x) \in\right] x_{r}, y_{r}[\}, \\
& E_{3}(\sigma)=\left\{x \in E: \Pi_{\mathcal{C}}(x) \in\left[y_{1}, y_{r}\right]\right\}, \\
& E_{4}(\sigma)=\left\{x \in E: \Pi_{\mathcal{C}}(x) \in\left[x_{1}, x_{r}\right]\right\},
\end{aligned}
$$

and proceed to estimate the integrals

$$
\mathcal{E}_{i}(\sigma):=\int_{E_{i}(\sigma)} v\left\langle D u, D \psi_{\sigma}\right\rangle d x, \quad i=1, \ldots, 4 .
$$

To find an upper bound for $\mathcal{E}_{1}(\sigma)$, observe that $\left|E_{1}(\sigma)\right| \leq \sigma\left|y_{1}-x_{1}\right|$ and $D \psi_{\sigma}=$ $-e_{1} / \sigma$ on $E_{1}(\sigma)$. Therefore, recalling that $\operatorname{Du}\left(x_{0}\right)=e_{2}$,

$$
\begin{aligned}
\left|\mathcal{E}_{1}(\sigma)\right| & =\frac{1}{\sigma}\left|\int_{E_{1}(\sigma)} v\left\langle D u, e_{1}\right\rangle d x\right|=\frac{1}{\sigma}\left|\int_{E_{1}(\sigma)} v\left\langle D u-D u\left(x_{0}\right), e_{1}\right\rangle d x\right| \\
& \leq \frac{1}{\sigma}\left|E_{1}(\sigma)\right|\|v\|_{\infty, \mathcal{C}_{\sigma}}\left\|D u-D u\left(x_{0}\right)\right\|_{\infty, E_{1}(\sigma)} \\
& \leq\left|y_{1}-x_{1}\right|\|v\|_{\infty, \mathcal{C}_{\sigma}}\left\|D u-D u\left(x_{0}\right)\right\|_{\infty, E_{1}(\sigma)} .
\end{aligned}
$$


Moreover, since $u$ is continuously differentiable at every point $x \in] y_{0}, x_{0}[$ and satisfies $D u(x)=D u\left(x_{0}\right)$, we have

$$
\omega_{1}(\sigma):=\left\|D u-D u\left(x_{0}\right)\right\|_{\infty, E_{1}(\sigma)} \rightarrow 0 \quad \text { as } \sigma \downarrow 0 .
$$

Similarly,

$$
\left|\mathcal{E}_{2}(\sigma)\right| \leq\left|y_{r}-x_{r}\right|\|v\|_{\infty, \mathcal{C}_{\sigma}} \omega_{2}(\sigma),
$$

where $\omega_{2}(\sigma):=\left\|D u-D u\left(x_{0}\right)\right\|_{\infty, E_{2}(\sigma)} \rightarrow 0$ as $\sigma \downarrow 0$.

Next, to bound $\mathcal{E}_{3}(\sigma)$ we note that $E_{3}(\sigma) \subset B_{\rho}\left(y_{0}\right)$ for $\sigma>0$ small enough. So, in view of 23, $\left|\mathcal{E}_{3}(\sigma)\right| \leq \varepsilon\left|E_{3}(\sigma)\right| / \sigma$ because $\left|D \psi_{\sigma}\right| \leq 1 / \sigma$ and $|D u| \leq 1$. Since $\left|E_{3}(\sigma)\right| \leq 2 \sigma\left(\left|y_{1}-y_{r}\right|+2 \sigma\right)$, we finally get the estimate

$$
\left|\mathcal{E}_{3}(\sigma)\right| \leq 2 \varepsilon\left(\left|y_{1}-y_{r}\right|+2 \sigma\right) .
$$

The reasoning we need to estimate $\mathcal{E}_{4}(\sigma)$ is just slightly longer than the previous ones. Let us split $E_{4}(\sigma)$ in two parts, $E_{4}^{\prime}(\sigma)$ and $E_{4}^{\prime \prime}(\sigma)$, where

$$
\begin{aligned}
& E_{4}^{\prime}(\sigma)=\left\{x \in E_{4}(\sigma): \Pi_{\mathcal{C}}(x) \in\right] x_{1}, x_{r}[\} \\
& E_{4}^{\prime \prime}(\sigma)=\left\{x \in E_{4}(\sigma): \Pi_{\mathcal{C}}(x) \in\left\{x_{1}, x_{r}\right\}\right\}
\end{aligned}
$$

By choosing $\sigma>0$ so small that $E_{4}(\sigma) \subset B_{\rho}\left(x_{0}\right)$, we have $\left|D u-e_{2}\right| \leq 1 / 2$ a.e. in $E_{4}(\sigma)$ owing to 21). Therefore,

$$
\left\langle D u, D \psi_{\sigma}\right\rangle \leq\left\langle e_{2}, D \psi_{\sigma}\right\rangle+\frac{1}{2 \sigma} \leq-\frac{1}{2 \sigma} \quad \text { a.e. in } E_{4}^{\prime}(\sigma)
$$

because, on that set, $D \psi_{\sigma}=-e_{2} / \sigma$. Now, by 22,

$$
\begin{aligned}
\mathcal{E}_{4}(\sigma) & \leq \int_{E_{4}^{\prime}(\sigma)} v\left\langle D u, D \psi_{\sigma}\right\rangle d x+\frac{\left|E_{4}^{\prime \prime}(\sigma)\right|}{\sigma}\|v\|_{\infty, \mathcal{C}_{\sigma}} \\
& \leq-\frac{1}{2 \sigma} \frac{v\left(x_{0}\right)}{2}\left|E_{4}^{\prime \prime}(\sigma)\right|+2 \pi \sigma\|v\|_{\infty, \mathcal{C}_{\sigma}} \\
& \leq-\frac{v\left(x_{0}\right)}{4}\left|x_{1}-x_{r}\right|+2 \pi \sigma\|v\|_{\infty, \mathcal{C}_{\sigma}} .
\end{aligned}
$$

Now, plugging estimates 26-29 into 25, we obtain

$$
\begin{aligned}
0 \leq \int_{\Omega} f \psi_{\sigma} d x \leq & 2 \varepsilon\left(\left|y_{1}-y_{r}\right|+2 \sigma\right)-\frac{v\left(x_{0}\right)}{4}\left|x_{1}-x_{r}\right| \\
& +\|v\|_{\infty, \mathcal{C}_{\sigma}}\left[\left|y_{1}-x_{1}\right| \omega_{1}(\sigma)+\left|y_{r}-x_{r}\right| \omega_{2}(\sigma)+2 \pi \sigma\right]
\end{aligned}
$$

Hence, letting $\sigma \downarrow 0$,

$$
0 \leq 2 \varepsilon\left|y_{1}-y_{r}\right|-\frac{v\left(x_{0}\right)}{4}\left|x_{1}-x_{r}\right|
$$


Since $\left|D u\left(x_{r}\right)-D u\left(x_{0}\right)\right| \leq \varepsilon r+L\left|x_{r}-x_{0}\right|$, where $L=\left\|D^{2} u\left(x_{0}\right)\right\|$, we have

$$
\begin{aligned}
\left|y_{1}-y_{r}\right| & =\left|x_{r}-\right| x_{0}-y_{1}\left|D u\left(x_{r}\right)-\left(x_{0}-\left|x_{0}-y_{1}\right| D u\left(x_{0}\right)\right)\right| \\
& \leq\left|x_{0}-y_{1}\right|\left(\varepsilon r+L\left|x_{r}-x_{0}\right|\right)+\left|x_{r}-x_{0}\right| .
\end{aligned}
$$

But $\left|x_{r}-x_{0}\right| \leq r$ and, by 24](iii), $\left|x_{1}-x_{r}\right| \geq r / 2$. So,

$$
\varepsilon r+L\left|x_{r}-x_{0}\right| \leq 2(\varepsilon+L)\left|x_{1}-x_{r}\right|
$$

and

$$
\left|y_{1}-y_{r}\right| \leq 2\left[1+\left|x_{0}-y_{1}\right|(\varepsilon+L)\right]\left|x_{1}-x_{r}\right| .
$$

Combining 30, and 31, we obtain

$$
0 \leq\left\{4 \varepsilon\left[1+\left|x_{0}-y_{1}\right|(\varepsilon+L)\right]-\frac{v\left(x_{0}\right)}{4}\right\}\left|x_{1}-x_{r}\right|,
$$

which contradicts 20). We have reached the contradiction assuming $u\left(x_{0}\right)<d\left(x_{0}\right)$. So, $u \equiv d$ and the proof is complete.

Our next task is to show that $v$ is given by the representation formula (4). We will do this in the next two propositions: the first one computes $v$ away from the singular set, the second one on $\bar{\Sigma}$.

Proposition 4.2. Let $(d, v)$ be a solution of system $(2)$. Then, for any $z_{0} \in \Omega \backslash \bar{\Sigma}$ and $\theta \in\left(0, \tau\left(z_{0}\right)\right)$, we have

$$
\begin{aligned}
& v\left(z_{0}\right)-\frac{1-\left(d\left(z_{0}\right)+\theta\right) \kappa\left(z_{0}\right)}{1-d\left(z_{0}\right) \kappa\left(z_{0}\right)} v\left(z_{0}+\theta \operatorname{Dd}\left(z_{0}\right)\right) \\
&=\int_{0}^{\theta} f\left(z_{0}+t \operatorname{Dd}\left(z_{0}\right)\right) \frac{1-\left(d\left(z_{0}\right)+t\right) \kappa\left(z_{0}\right)}{1-d\left(z_{0}\right) \kappa\left(z_{0}\right)} d t .
\end{aligned}
$$

Proof. Let $z_{0} \in \Omega \backslash \bar{\Sigma}, \theta \in\left(0, \tau\left(z_{0}\right)\right)$ and set $x_{0}=z_{0}+\theta D d\left(z_{0}\right)$. Notice that $\left[z_{0}, x_{0}\right] \subset$ $\Omega \backslash \bar{\Sigma}$ and $D d(z)=D d\left(z_{0}\right)$ for $z \in\left[z_{0}, x_{0}\right]$ by Proposition 2.2 (b).

Let us use-once again - a coordinate system that simplifies the notation: we set $e_{2}=$ $\operatorname{Dd}\left(z_{0}\right)$ and choose $e_{1}$ such that $\left\{e_{1}, e_{2}\right\}$ is a positively oriented orthonormal basis of $\mathbb{R}^{2}$. Also, fix $r>0$ so small that $x_{r}:=x_{0}+r e_{1} \notin \bar{\Sigma}$ and $\left\langle D d\left(x_{r}\right), e_{2}\right\rangle>0$. Let then $\bar{t}>0$ be such that the point $z_{r}:=x_{r}-\bar{t} D d\left(x_{r}\right)$ satisfies $\left\langle z_{r}-z_{0}, e_{2}\right\rangle=0$. We note that $\bar{t}$ is given by

$$
\bar{t}=\frac{\left\langle x_{r}-z_{0}, e_{2}\right\rangle}{\left\langle D d\left(x_{r}\right), e_{2}\right\rangle}=\frac{\left|x_{0}-z_{0}\right|}{\left\langle D d\left(x_{r}\right), e_{2}\right\rangle} .
$$

Finally, let us possibly reduce $r>0$ to ensure that the domain $D_{r}:=\operatorname{co}\left\{x_{0}, x_{r}, z_{r}, z_{0}\right\}$ be contained in $\Omega \backslash \bar{\Sigma}$ and $d$ be of class $\mathcal{C}^{2}$ in a neighbourhood of $D_{r}$.

Integrating by parts the equation $-\operatorname{div}(v D d)=f$ on $D_{r}$, we obtain

$$
\int_{D_{r}} f d x=-\int_{\partial D_{r}} v\langle D d, v\rangle d \mathcal{H}^{1}
$$


where $v$ is the outward unit normal to $\partial D_{r}$. The above right-hand side amounts to

$$
\int_{\partial D_{r}} v\langle D d, v\rangle d \mathcal{H}^{1}=\int_{\left[x_{0}, x_{r}\right]} v\left\langle D d, e_{2}\right\rangle d \mathcal{H}^{1}+\int_{\left[z_{0}, z_{r}\right]} v\left\langle D d,-e_{2}\right\rangle d \mathcal{H}^{1}
$$

because

$$
\int_{\left[z_{0}, x_{0}\right]} v\langle D d, v\rangle d \mathcal{H}^{1}=\int_{\left[z_{0}, x_{0}\right]} v\left\langle e_{2},-e_{1}\right\rangle d \mathcal{H}^{1}=0
$$

and, similarly, $\langle D d, v\rangle=0$ on $\left[z_{r}, x_{r}\right]$. Moreover, we have

$$
\int_{D_{r}} f d x=\int_{0}^{\left|z_{0}-x_{0}\right|} d t \int_{0}^{l_{t}} f\left(z_{0}+t e_{2}+s e_{1}\right) d s,
$$

where

$$
l_{t}=\left(1-\frac{t}{\left|z_{0}-x_{0}\right|}\right)\left|z_{0}-z_{r}\right|+\frac{t}{\left|z_{0}-x_{0}\right|}\left|x_{0}-x_{r}\right| .
$$

Our next step will be to compute $\lim _{r \downarrow 0} \frac{1}{r} \int_{D_{r}} f d x$. Aiming at this, let us recall that, in view of Proposition 2.6 .

$$
D^{2} d\left(x_{0}\right)=\gamma_{0}\left(e_{1} \otimes e_{1}\right), \quad \text { where } \quad \gamma_{0}=-\frac{\kappa\left(x_{0}\right)}{1-\kappa\left(x_{0}\right) d\left(x_{0}\right)} .
$$

Hence,

$$
\frac{1}{r} \frac{\left\langle D d\left(x_{r}\right), e_{1}\right\rangle}{\left\langle D d\left(x_{r}\right), e_{2}\right\rangle}=\frac{1}{r} \frac{\left\langle D d\left(x_{0}\right)+r D^{2} d\left(x_{0}\right) e_{1}+\mathrm{o}(r), e_{1}\right\rangle}{\left\langle D d\left(x_{0}\right)+r D^{2} d\left(x_{0}\right) e_{1}+\mathrm{o}(r), e_{2}\right\rangle}=\frac{\gamma_{0}+\epsilon(r)}{1+\epsilon(r)},
$$

where $\epsilon(r) \rightarrow 0$ as $r \downarrow 0$. Since

$$
\frac{\left|z_{0}-z_{r}\right|}{r}=1-\left|x_{0}-z_{0}\right| \frac{1}{r} \frac{\left\langle D d\left(x_{r}\right), e_{1}\right\rangle}{\left\langle D d\left(x_{r}\right), e_{2}\right\rangle}=1-\left|x_{0}-z_{0}\right| \frac{\gamma_{0}+\epsilon(r)}{1+\epsilon(r)},
$$

we obtain

$$
\begin{aligned}
\lim _{r \rightarrow 0^{+}} \frac{l_{t}}{r} & =\left(1-\frac{t}{\left|z_{0}-x_{0}\right|}\right)\left(1-\gamma_{0}\left|x_{0}-z_{0}\right|\right)+\frac{t}{\left|z_{0}-x_{0}\right|} \\
& =1-\gamma_{0}\left|x_{0}-z_{0}\right|+t \gamma_{0} .
\end{aligned}
$$

Therefore, in view of (35), we conclude that

$$
\lim _{r \downarrow 0} \frac{1}{r} \int_{D_{r}} f d x=\int_{0}^{\left|z_{0}-x_{0}\right|} f\left(z_{0}+t e_{2}\right)\left(1-\gamma_{0}\left|x_{0}-z_{0}\right|+t \gamma_{0}\right) d t .
$$

We now turn to the evaluation of $\lim _{r \downarrow 0} \frac{1}{r} \int_{\partial D_{r}} v\langle D d, v\rangle$. Since $D d$ is continuous at $x_{0}$ and $\operatorname{Dd}\left(x_{0}\right)=e_{2}$, we have

$$
\lim _{r \downarrow 0} \frac{1}{r} \int_{\left[x_{0}, x_{r}\right]} v\left\langle D d, e_{2}\right\rangle d \mathcal{H}^{1}=v\left(x_{0}\right) .
$$


A similar continuity argument and 36 show that

$$
\lim _{r \downarrow 0} \frac{1}{r} \int_{\left[z_{0}, z_{r}\right]} v\left\langle D d,-e_{2}\right\rangle d \mathcal{H}^{1}=-v\left(z_{0}\right)\left(1-\gamma_{0}\left|x_{0}-z_{0}\right|\right) .
$$

Then, recalling (33), 34) and (37), we conclude that

$$
\begin{aligned}
\lim _{r \downarrow 0}-\frac{1}{r} \int_{\partial D_{r}} v\langle D d, v\rangle d \mathcal{H}^{1} & =v\left(z_{0}\right)\left(1-\gamma_{0}\left|x_{0}-z_{0}\right|\right)-v\left(x_{0}\right) \\
& =\int_{0}^{\left|z_{0}-x_{0}\right|} f\left(z_{0}+t e_{2}\right)\left(1-\gamma_{0}\left|x_{0}-z_{0}\right|+t \gamma_{0}\right) d t
\end{aligned}
$$

whence, since $\left|z_{0}-x_{0}\right|=\theta$,

$$
v\left(z_{0}\right)-\frac{v\left(x_{0}\right)}{1-\gamma_{0} \theta}=\int_{0}^{\theta} f\left(z_{0}+t e_{2}\right)\left(1+\frac{t \gamma_{0}}{1-\gamma_{0} \theta}\right) d t .
$$

Finally, recalling the definition of $\gamma_{0}$ and using the equality $d\left(x_{0}\right)=d\left(z_{0}\right)+\theta$, we have

$$
1-\gamma_{0} \theta=1+\frac{\kappa\left(x_{0}\right) \theta}{1-d\left(x_{0}\right) \kappa\left(x_{0}\right)}=\frac{1-d\left(z_{0}\right) \kappa\left(x_{0}\right)}{1-d\left(x_{0}\right) \kappa\left(x_{0}\right)}
$$

and

$$
\frac{\gamma_{0}}{1-\gamma_{0} \theta}=-\frac{\kappa\left(x_{0}\right)}{1-d\left(z_{0}\right) \kappa\left(x_{0}\right)} .
$$

In view of the above identities and of the fact that $\kappa\left(x_{0}\right)=\kappa\left(z_{0}\right), 38$ can be recast as

$$
v\left(z_{0}\right)-\frac{1-d\left(x_{0}\right) \kappa\left(z_{0}\right)}{1-d\left(z_{0}\right) \kappa\left(z_{0}\right)} v\left(x_{0}\right)=\int_{0}^{\theta} f\left(z_{0}+t e_{2}\right) \frac{1-\left(d\left(z_{0}\right)+t\right) \kappa\left(z_{0}\right)}{1-d\left(z_{0}\right) \kappa\left(z_{0}\right)} d t .
$$

This is the conclusion.

The following result is reminiscent of [16, Proposition 7.1].

Proposition 4.3. If $(d, v)$ is a solution of system (2), then $v=0$ on $\bar{\Sigma}$.

Proof. Assume, first, that $\Sigma$ is a singleton, say $\left\{x_{0}\right\}$. Then, by a classical result of Motzkin's [26] (see also Remark 2.11), $\Omega$ is the disk $B_{R}\left(x_{0}\right)$ with $R=d\left(x_{0}\right)$. Integrating the equation $-\operatorname{div}(v D d)=f$ on $B_{r}\left(x_{0}\right)$, for $0<r<R$, gives

$$
\int_{B_{r}\left(x_{0}\right)} f d x=-\int_{\partial B_{r}\left(x_{0}\right)} v\langle D d, v\rangle d \mathcal{H}^{1},
$$

where $v$ is the outward unit normal to $\partial B_{r}\left(x_{0}\right)$. Since $\langle D d, v\rangle=-1$, we have

$$
0=\lim _{r \downarrow 0} \frac{1}{r} \int_{B_{r}\left(x_{0}\right)} f d x=\lim _{r \downarrow 0} \frac{1}{r} \int_{\partial B_{r}\left(x_{0}\right)} v d \mathcal{H}^{1}=2 \pi v\left(x_{0}\right) .
$$

Thus, $v\left(x_{0}\right)=0$. 
Suppose, next, that $\Sigma$ is not a singleton. Then, again by Remark 2.11, the set $\Sigma^{1}$ of singular points with magnitude 1 is dense in $\Sigma$. Since $v$ is continuous, it suffices to prove that $v$ vanishes on $\Sigma^{1}$. So, let $x_{0} \in \Sigma^{1}$ and $D^{+} d\left(x_{0}\right)=\left[p_{0}, q_{0}\right]$ with $p_{0} \neq q_{0}$. Then, by Proposition 2.10, there exists a Lipschitz arc $\boldsymbol{x}:[0, \eta] \rightarrow \Sigma$ such that $\boldsymbol{x}(0)=x_{0}$, $\dot{\boldsymbol{x}}(0) \neq 0$, and

$$
\left\langle\dot{\boldsymbol{x}}(0), p_{0}-q_{0}\right\rangle=0 .
$$

Moreover, $\boldsymbol{x}\left(s_{n}\right) \in \Sigma^{1}$ for some sequence $s_{n} \downarrow 0$, and

$$
D^{+} d\left(\boldsymbol{x}\left(s_{n}\right)\right)=\left[p_{n}, q_{n}\right] \quad \text { with } \quad p_{n} \rightarrow p_{0}, q_{n} \rightarrow q_{0} .
$$

Since $\Sigma$ has Lebesgue measure zero, we have, by Fubini's Theorem,

$$
\mathcal{H}^{1}\left(\left[x_{0}-\alpha s_{n} p_{0}, \boldsymbol{x}\left(s_{n}\right)-\alpha s_{n} p_{n}\right] \cap \Sigma\right)=0 \quad \text { for a.e. } \alpha \in[1,2],
$$

provided $n$ is sufficiently large. Let $\alpha_{n} \in[1,2]$ be such that

$$
\mathcal{H}^{1}\left(\left[x_{0}-\alpha_{n} s_{n} p_{0}, \boldsymbol{x}\left(s_{n}\right)-\alpha_{n} s_{n} p_{n}\right] \cap \Sigma\right)=0 .
$$

In the same way, let $\beta_{n} \in[1,2]$ be such that

$$
\mathcal{H}^{1}\left(\left[x_{0}-\beta_{n} s_{n} q_{0}, \boldsymbol{x}\left(s_{n}\right)-\beta_{n} s_{n} q_{n}\right] \cap \Sigma\right)=0 .
$$

Set, for every $n \in \mathbb{N}$,

$$
\left.I_{p}^{n}:=\right] x_{0}-\alpha_{n} s_{n} p_{0}, \boldsymbol{x}\left(s_{n}\right)-\alpha_{n} s_{n} p_{n}\left[, \quad I_{q}^{n}:=\right] x_{0}-\beta_{n} s_{n} q_{0}, \boldsymbol{x}\left(s_{n}\right)-\beta_{n} s_{n} q_{n}[.
$$

Now, for $n \in \mathbb{N}$ large enough define the domain

$$
D_{n}:=\operatorname{co}\left(\left[x_{0}, \boldsymbol{x}\left(s_{n}\right)\right] \cup \overline{I_{p}^{n}}\right) \cup \operatorname{co}\left(\left[x_{0}, \boldsymbol{x}\left(s_{n}\right)\right] \cup \overline{I_{q}^{n}}\right)
$$

(the bar denotes closure) and consider, for $\sigma>0$, the function

$$
\psi_{\sigma}^{n}(x)=\left[1-\frac{1}{\sigma} d_{D_{n}}(x)\right]_{+}, \quad x \in \Omega .
$$

Notice that, for $n$ large enough, $\psi_{\sigma}^{n} \in W_{\mathrm{c}}^{1, \infty}(\Omega)$. Therefore, using $\psi_{\sigma}^{n}$ as test function for the equation $-\operatorname{div}(v D d)=f$, we have

$$
\int_{\Omega} f \psi_{\sigma}^{n} d x=\int_{\Omega} v\left\langle D d, D \psi_{\sigma}^{n}\right\rangle d x .
$$

In order to estimate the right-hand side, observe that the support of $D \psi_{\sigma}^{n}$ is the closure of the set $A^{n}(\sigma):=\left\{x \in \Omega \backslash D_{n}: d_{D_{n}}(x)<\sigma\right\}$. This set can be represented as the disjoint union $A_{p}^{n}(\sigma) \cup A_{q}^{n}(\sigma) \cup \widetilde{A}^{n}(\sigma)$, where

$$
A_{p}^{n}(\sigma)=\left\{x \in A(\sigma): \Pi_{D_{n}}(x) \in I_{p}^{n}\right\}, \quad A_{q}^{n}(\sigma)=\left\{x \in A(\sigma): \Pi_{D_{n}}(x) \in I_{q}^{n}\right\} .
$$


Then the gradient of $d_{D_{n}}$ is constant on $A_{p}^{n}(\sigma)$, say $D d_{D_{n}} \equiv v_{p}^{n}$. Similarly, $D d_{D_{n}} \equiv v_{q}^{n}$ on $A_{q}^{n}(\sigma)$. Now, observe that

$$
\begin{aligned}
& -\int_{\Omega} f \psi_{\sigma} d x \\
& =\int_{A_{p}^{n}(\sigma)} \frac{v}{\sigma}\left\langle D d, v_{p}^{n}\right\rangle d x+\int_{A_{q}^{n}(\sigma)} \frac{v}{\sigma}\left\langle D d, v_{q}^{n}\right\rangle d x+\int_{\widetilde{A}^{n}(\sigma)} v\left\langle D d, D \psi_{\sigma}^{n}\right\rangle d x .
\end{aligned}
$$

We will pass to the limit as $\sigma \downarrow 0$ in the above identity. We have

$$
\lim _{\sigma \downarrow 0} \int_{\Omega} f \psi_{\sigma}^{n} d x=\int_{D_{n}} f d x .
$$

Moreover, arguing as in the proof of Proposition 4.1. we find

$$
\lim _{\sigma \downarrow 0} \int_{\widetilde{A}^{n}(\sigma)} v\left\langle D d, D \psi_{\sigma}^{n}\right\rangle d x=0 .
$$

In order to estimate the term

$$
\int_{A_{p}^{n}(\sigma)} \frac{v}{\sigma}\left\langle D d, v_{p}^{n}\right\rangle d x=\frac{1}{\sigma} \int_{I_{p}^{n}} d \mathcal{H}^{1}(y) \int_{0}^{\sigma} v\left(y+t v_{p}^{n}\right)\left\langle D d\left(y+t v_{p}^{n}\right), v_{p}^{n}\right\rangle d t,
$$

recall that $\mathcal{H}^{1}\left(I_{p}^{n} \cap \Sigma\right)=0$, and so $D d$ is continuous at $\mathcal{H}^{1}$-almost every point of $I_{p}^{n}$. Therefore,

$$
\lim _{\sigma \downarrow 0} \int_{A_{p}^{n}(\sigma)} \frac{v}{\sigma}\left\langle D d, v_{p}^{n}\right\rangle d x=\int_{I_{p}^{n}} v(y)\left\langle D d(y), v_{p}^{n}\right\rangle d \mathcal{H}^{1}(y) .
$$

Similarly,

$$
\lim _{\sigma \downarrow 0} \int_{A_{q}^{n}(\sigma)} \frac{v}{\sigma}\left\langle D d, v_{q}^{n}\right\rangle d x=\int_{I_{q}^{n}} v(y)\left\langle\operatorname{Dd}(y), v_{q}^{n}\right\rangle d \mathcal{H}^{1}(y) .
$$

Thus, passing to the limit as $\sigma \downarrow 0$ in (41), we conclude that

$$
-\int_{D_{n}} f=\int_{I_{p}^{n}} v(y)\left\langle D d(y), v_{p}^{n}\right\rangle d \mathcal{H}^{1}(y)+\int_{I_{q}^{n}} v(y)\left\langle D d(y), v_{q}^{n}\right\rangle d \mathcal{H}^{1}(y) .
$$

Our final step will be to divide both sides of (42) by $s_{n}$ and to take the limit as $n \rightarrow \infty$. For this we need two preliminary remarks. The first one is that, for every sequence $\left\{y_{n}\right\}_{n}$ such that $y_{n} \in I_{p}^{n}$ and $d$ is differentiable at $y_{n}, \operatorname{Dd}\left(y_{n}\right)$ converges to $p_{0}$ as $n \rightarrow \infty$. For let $\lambda_{n} \in[0,1]$ be such that

$$
\begin{aligned}
y_{n} & =\lambda_{n}\left(x_{0}-\alpha_{n} s_{n} p_{0}\right)+\left(1-\lambda_{n}\right)\left(\boldsymbol{x}\left(s_{n}\right)-\alpha_{n} s_{n} p_{n}\right) \\
& =\lambda_{n}\left(x_{0}-\alpha_{n} s_{n} p_{0}\right)+\left(1-\lambda_{n}\right)\left(x_{0}+s_{n} \dot{\boldsymbol{x}}(0)+\mathrm{o}\left(s_{n}\right)-\alpha_{n} s_{n} p_{n}\right)
\end{aligned}
$$


and suppose $\lambda_{n} \rightarrow \lambda^{*} \in[0,1]$ and $\alpha_{n} \rightarrow \alpha^{*} \in[1,2]$ as $n \rightarrow \infty$ (which always holds, up to subsequences). Then

$$
\lim _{n \rightarrow \infty} \frac{y_{n}-x_{0}}{s_{n}}=-\alpha^{*} \lambda^{*} p_{0}+\left(1-\lambda^{*}\right) \dot{\boldsymbol{x}}(0)=: \theta^{*} .
$$

But $\min \left\{\left\langle p, \theta^{*}\right\rangle: p \in D^{+} d\left(x_{0}\right)\right\}$ is attained at $p_{0}$, since, in view of 39 , $\langle\dot{\boldsymbol{x}}(0), p\rangle=$ $\left\langle\dot{\boldsymbol{x}}(0), p_{0}\right\rangle$ for every $p \in\left[p_{0}, q_{0}\right]$. Thus, by [3, Theorem 2.1], $D d\left(y_{n}\right) \rightarrow p_{0}$ as claimed.

The second remark we need to proceed with our computation is that

$$
\lim _{n \rightarrow \infty} v_{p}^{n}=-\frac{p_{0}-q_{0}}{\left|p_{0}-q_{0}\right|} .
$$

Indeed, by definition,

$$
\left\langle v_{p}^{n}, \boldsymbol{x}\left(s_{n}\right)-\alpha_{n} s_{n} p_{n}-\left(x_{0}-\alpha_{n} s_{n} p_{0}\right)\right\rangle=0,
$$

where

$$
\boldsymbol{x}\left(s_{n}\right)-x_{0}+\alpha_{n} s_{n}\left(p_{0}-p_{n}\right)=s_{n} \dot{\boldsymbol{x}}(0)+\mathrm{o}\left(s_{n}\right)
$$

in view of 40p. Thus, $v_{p}^{n}$ is nearly orthogonal to $\dot{\boldsymbol{x}}(0)$, and so

$$
v_{p}^{n}=\rho_{0}\left(p_{0}-q_{0}\right)+\varepsilon_{n} \quad \text { with } \quad \lim _{n \rightarrow \infty} \varepsilon_{n}=0 \quad \text { and } \quad\left|\rho_{0}\right|=\frac{1}{\left|p_{0}-q_{0}\right|} .
$$

Moreover, $\left\langle v_{p}^{n}, p_{0}\right\rangle \leq 0$ for $n$ large enough, because $v_{p}^{n}$ is an outward normal to the set $\operatorname{co}\left(\left[x_{0}, \boldsymbol{x}\left(s_{n}\right)\right] \cup \overline{I_{p}^{n}}\right)$ at the point $x_{0}-\alpha_{n} s_{n} p_{0}$ and $x_{0}$ belongs to that set. Therefore, $\rho_{0}<0$ and (43) follows.

We are now ready for our final step. Dividing both sides of (42) by $s_{n}$ and taking the limit as $n \rightarrow \infty$, we obtain

$$
0=\lim _{n \rightarrow \infty} \frac{1}{s_{n}}\left\{\int_{I_{p}^{n}} v(y)\left\langle D d(y), v_{p}^{n}\right\rangle d \mathcal{H}^{1}(y)+\int_{I_{q}^{n}} v(y)\left\langle D d(y), v_{q}^{n}\right\rangle d \mathcal{H}^{1}(y)\right\} .
$$

Since $\mathcal{H}^{1}\left(I_{p}^{n}\right)=\left|\boldsymbol{x}\left(s_{n}\right)-\alpha_{n} s_{n} p_{n}-\left(x_{0}-\alpha_{n} s_{n} p_{0}\right)\right|=s_{n}|\dot{\boldsymbol{x}}(0)|+\mathrm{o}\left(s_{n}\right)$ on account of [44, we have

$$
\lim _{n \rightarrow \infty} \frac{1}{s_{n}} \int_{I_{p}^{n}} v(y)\left\langle D d(y), v_{p}^{n}\right\rangle d \mathcal{H}^{1}(y)=-v\left(x_{0}\right)|\dot{\boldsymbol{x}}(0)|\left\langle p_{0}, \frac{p_{0}-q_{0}}{\left|p_{0}-q_{0}\right|}\right\rangle .
$$

By a similar argument,

$$
\lim _{n} \frac{1}{s_{n}} \int_{I_{q}^{n}} v(y)\left\langle D d(y), v_{q}^{n}\right\rangle d \mathcal{H}^{1}(y)=v\left(x_{0}\right)|\dot{\boldsymbol{x}}(0)|\left\langle q_{0}, \frac{p_{0}-q_{0}}{\left|p_{0}-q_{0}\right|}\right\rangle .
$$

Thus,

$$
\begin{aligned}
0 & =v\left(x_{0}\right)|\dot{\boldsymbol{x}}(0)|\left\{-\left\langle p_{0}, \frac{p_{0}-q_{0}}{\left|p_{0}-q_{0}\right|}\right\rangle+\left\langle q_{0}, \frac{p_{0}-q_{0}}{\left|p_{0}-q_{0}\right|}\right\rangle\right\} \\
& =-v\left(x_{0}\right)|\dot{\boldsymbol{x}}(0)|\left|p_{0}-q_{0}\right| .
\end{aligned}
$$

Since $\dot{\boldsymbol{x}}(0) \neq 0$ and $p_{0} \neq q_{0}$, we conclude that $v\left(x_{0}\right)=0$. 
We are now ready to complete the proof of our main result.

Proof of Theorem 1.1 (uniqueness). Let $(u, v)$ be a solution of system (2). Then $u \equiv d$ in $\Omega_{v}:=\{x \in \Omega: v(x)>0\}$ by Proposition 4.1 In particular, $(d, v)$ is also a solution of (2). So, owing to Proposition 4.3, $v=0$ on $\bar{\Sigma}$. Now, let $x \in \Omega \backslash \bar{\Sigma}$. In view of Proposition 4.2 , we have

$v(x)-\frac{1-(d(x)+\theta) \kappa(x)}{1-d(x) \kappa(x)} v(x+\theta D d(x))=\int_{0}^{\theta} f(x+t D d(x)) \frac{1-(d(x)+t) \kappa(x)}{1-d(x) \kappa(x)} d t$

for each $\theta \in(0, \tau(x))$. Since $v$ is continuous and vanishes on $\bar{\Sigma}$, the left-hand side above converges to $v(x)$ as $\theta \uparrow \tau(x)$. So, $v(x)$ coincides with $v^{f}(x)$, given by 4 , and the proof is complete.

\section{A. Appendix: Proof of Theorem 2.12}

We already know that the normal distance $\tau$, defined in 77 , is continuous in $\bar{\Omega}$ (see Proposition 2.14). In this section we will prove that if $\Omega$ has a $\mathcal{C}^{2,1}$ boundary, then $\tau$ is also Lipschitz continuous on $\partial \Omega$ (Theorem 2.12). The main step of the proof is the following preliminary result.

Lemma A.1. Let $\Omega$ be a bounded domain in $\mathbb{R}^{2}$ with boundary of class $\mathcal{C}^{2,1}$. Then every $x \in \partial \Omega$ has a neighbourhood, $U$, such that

$$
\tau(y) \leq \tau(x)+K(\operatorname{diam} \Omega)^{2}|y-x| \quad \forall y \in \partial \Omega \cap U,
$$

where

$$
K=\sup _{\substack{x, y \in \partial \Omega \\ x \neq y}} \max \left\{\frac{|\kappa(y)-\kappa(x)|}{|y-x|}, \frac{\left|D d(y)-D d(x)-D^{2} d(x)(y-x)\right|}{|y-x|^{2}}\right\} .
$$

Proof. Let $x \in \partial \Omega$ be fixed. We will analyse, first, the simpler case $\tau(x) \kappa(x)=1$. Recalling that $\tau(x) \leq \operatorname{diam} \Omega / 2$, we have $\kappa(x) \geq 2 / \operatorname{diam} \Omega$. Let $U$ be an open neighbourhood of $x$ such that $k(y)>1 / \operatorname{diam} \Omega$ for every $y \in U$. Then, for every $y \in \partial \Omega \cap U$,

$$
\tau(y) \leq \frac{1}{\kappa(y)} \leq \frac{1}{\kappa(x)}+\frac{\kappa(y)-\kappa(x)}{\kappa(y) \kappa(x)} \leq \tau(x)+\frac{K}{2}(\operatorname{diam} \Omega)^{2}|y-x|
$$

and 45 is proved.

Now, suppose $\tau(x) \kappa(x)<1$ and define $\bar{x}=x+\tau(x) \operatorname{Dd}(x)$. We claim that $\operatorname{Dd}(x)$ must be isolated in $D^{*} d(\bar{x})$. For suppose $D d(x)=\lim _{k} p_{k}$ for some sequence $\left\{p_{k}\right\}$ in $D^{*} d(\bar{x})$ satisfying $p_{k} \neq D d(x)$ for every $k$. Then $p_{k}=D d\left(x_{k}\right)$, where $x_{k}=\bar{x}-$ $d(\bar{x}) p_{k} \neq \bar{x}-d(\bar{x}) D d(x)=x$ is a sequence of boundary points converging to $x$. We can also assume, without loss of generality, that $\left(x_{k}-x\right) /\left|x_{k}-x\right|$ converges to some unit vector $\theta$. Hence,

$$
\theta=\lim _{k \rightarrow \infty} \frac{x_{k}-x}{\left|x_{k}-x\right|}=-d(\bar{x}) \lim _{k \rightarrow \infty} \frac{D d\left(x_{k}\right)-D d(x)}{\left|x_{k}-x\right|}=-d(\bar{x}) D^{2} d(x) \theta .
$$


Therefore, recalling that the nonzero eigenvalue of $D^{2} d(x)$ is given by $-\kappa(x)$, we obtain $-\kappa(x)=-1 / d(\bar{x})=-1 / \tau(x)$, contrary to $\tau(x) \kappa(x)<1$. So, our claim is proved.

Hereafter, we denote by $\mathcal{R}$ the rotation matrix

$$
\mathcal{R}=\left(\begin{array}{rr}
0 & -1 \\
1 & 0
\end{array}\right)
$$

and by $\left\{e_{1}, e_{2}\right\}$ the orthonormal basis of $\mathbb{R}^{2}$ given by

$$
e_{1}=\mathcal{R}^{-1} D d(x), \quad e_{2}=\operatorname{Dd}(x) .
$$

We split the reasoning into several steps.

Step 1: construction of a singular arc. We want to construct a Lipschitz $\operatorname{arc} x:[0, \eta] \rightarrow$ $\Omega$ such that

$$
\boldsymbol{x}(0)=\bar{x}, \quad|\dot{\boldsymbol{x}}(0)|=1, \quad\left\langle\dot{\boldsymbol{x}}(0), e_{1}\right\rangle>0, \quad \boldsymbol{x}(s) \in \Sigma \quad \forall s \in[0, \eta] .
$$

Suppose, first, $\bar{x} \in \Sigma^{2}$. Since $e_{2}$ is isolated in $D^{*} d(\bar{x})$, there are two distinct vectors $p_{1}, p_{2} \in D^{*} d(\bar{x})$ such that the segments $\left[p_{1}, e_{2}\right]$ and $\left[p_{2}, e_{2}\right]$ are contained in $\partial D^{+} d(\bar{x})$. Let $n_{1}$ and $n_{2}$ be outward unit normals to $D^{+} d(\bar{x})$ exposing the faces [ $\left.p_{1}, e_{2}\right]$ and $\left[p_{2}, e_{2}\right]$ respectively, i.e.

$$
\max _{p \in D^{+} d(\bar{x})}\left\langle p, n_{i}\right\rangle=\left\langle p_{i}, n_{i}\right\rangle=\left\langle e_{2}, n_{i}\right\rangle, \quad i=1,2 .
$$

We claim that

$$
e_{2}=\lambda_{1} n_{1}+\lambda_{2} n_{2}
$$

for suitable numbers $\lambda_{1}, \lambda_{2}>0$. Indeed, the normal cone to $D^{+} d(\bar{x})$ at $e_{2}$ is generated by $\left\{n_{1}, n_{2}\right\}$. Since $e_{2}$ belongs to this cone, $e_{2}=\lambda_{1} n_{1}+\lambda_{2} n_{2}$ with $\lambda_{1}, \lambda_{2} \geq 0$. If $\lambda_{1}=0$, then $\lambda_{2}=1$ and $e_{2}=n_{2}$. Therefore, $\left\langle p_{2}, n_{2}\right\rangle=\left\langle e_{2}, n_{2}\right\rangle=1$, which implies $p_{2}=n_{2}=e_{2}$, contrary to the definition of $p_{2}$. So, $\lambda_{1}>0$. Similarly, $\lambda_{2}>0$; our claim is thus proved.

Now, observe that, on account of 477, $0=\lambda_{1}\left\langle n_{1}, e_{1}\right\rangle+\lambda_{2}\left\langle n_{2}, e_{1}\right\rangle$. So, either $\left\langle n_{1}, e_{1}\right\rangle$ $<0$ or $\left\langle n_{2}, e_{1}\right\rangle<0$. Suppose $\left\langle n_{1}, e_{1}\right\rangle<0$, and apply Proposition 2.10 to the face [ $\left.p_{1}, e_{2}\right]$ of $D^{+} d(\bar{x})$, with normal $n_{1}$, to construct a Lipschitz arc $x:[0, \eta] \rightarrow \Omega$ such that

$$
\boldsymbol{x}(0)=\bar{x}, \quad \dot{\boldsymbol{x}}(0)=-n_{1}, \quad \boldsymbol{x}(s) \in \Sigma \quad \forall s \in[0, \eta] .
$$

Since $\left\langle n_{1}, e_{1}\right\rangle<0$, we have $\left\langle\dot{\boldsymbol{x}}(0), e_{1}\right\rangle>0$, which proves (46).

To complete the proof of this step it suffices to note that the case $\bar{x} \in \Sigma^{1}$ can be treated by a similar-yet simpler-argument.

Step 2: normals to $\partial \Omega$ do intersect the singular arc. We want to construct a neighbourhood $U$ of $x$ such that, for any boundary point $y \in \partial \Omega \cap U$ satisfying $\left\langle y-x, e_{1}\right\rangle>0$, there exist $s_{y}, \rho_{y}>0$ with

$$
\begin{gathered}
\boldsymbol{x}\left(s_{y}\right)=y+\rho_{y} D d(y), \\
\lim _{y \rightarrow x} s_{y}=0
\end{gathered}
$$

(where the limit is taken for $y \in \partial \Omega \cap U$ such that $\left\langle y-x, e_{1}\right\rangle>0$ ). 
Let $V$ be an open neighbourhood of $x$ such that $\partial \Omega \cap V$ is the trace of a regular curve $h \mapsto \boldsymbol{y}(h),-r<h<r$, with $\boldsymbol{y}(0)=x$ and $\dot{y}(0)=e_{1}$. Then $\boldsymbol{y}(h)=x+h e_{1}+\mathrm{o}(h)$. Moreover, for every $y \in \partial \Omega \cap V$ satisfying $\left\langle y-x, e_{1}\right\rangle>0$,

$$
\exists ! h_{y} \in(0, r) \quad \text { such that } \quad y=\boldsymbol{y}\left(h_{y}\right) .
$$

Now, consider the map $\phi:(0, r) \times[0, \eta] \rightarrow \mathbb{R}$ defined by

$$
\phi(h, s)=\langle\boldsymbol{x}(s)-\boldsymbol{y}(h), \mathcal{R} \operatorname{Dd}(\boldsymbol{y}(h))\rangle \quad(h \in(0, r), s \in[0, \eta]),
$$

where $\boldsymbol{x}$ is the singular arc of Step 1 . Since $D^{2} d(x)=-\kappa(x) e_{1} \otimes e_{1}$, we have

$$
\begin{aligned}
\mathcal{R} D d(\boldsymbol{y}(h)) & =\mathcal{R} D d(x)+\mathcal{R} D^{2} d(x)[\boldsymbol{y}(h)-x]+\mathrm{o}(|\boldsymbol{y}(h)-x|) \\
& =-e_{1}-h \kappa(x) e_{2}+\mathrm{o}(h) .
\end{aligned}
$$

Also,

$$
\boldsymbol{x}(s)-\boldsymbol{y}(h)=x+\tau(x) e_{2}+s \dot{\boldsymbol{x}}(0)-\left(x+h e_{1}\right)+\mathrm{o}(s)+\mathrm{o}(h) .
$$

Therefore,

$$
\begin{aligned}
\phi(h, s) & =\left\langle\tau(x) e_{2}+s \dot{\boldsymbol{x}}(0)-h e_{1},-e_{1}-h \kappa(x) e_{2}\right\rangle+\mathrm{o}(s)+\mathrm{o}(h) \\
& =h(1-\tau(x) \kappa(x))-s\left[\left\langle\dot{\boldsymbol{x}}(0), e_{1}\right\rangle+h \kappa(x)\left\langle\dot{\boldsymbol{x}}(0), e_{2}\right\rangle\right]+\mathrm{o}(s)+\mathrm{o}(h) .
\end{aligned}
$$

But $1-\tau(x) \kappa(x)>0$. So, $\phi(h, 0)>0$ for $h$ small enough, say $0<h<r_{0}$. Moreover, since $\left\langle\dot{\boldsymbol{x}}(0), e_{1}\right\rangle>0$, after possibly reducing $r_{0}$ we conclude that, for some $\bar{s} \in(0, \eta]$ and all $(h, s) \in\left(0, r_{0}\right) \times[0, \bar{s}]$,

$$
\phi(h, s) \leq-\frac{s}{2}\left\langle\dot{\boldsymbol{x}}(0), e_{1}\right\rangle+h(1-\tau(x) \kappa(x))+\mathrm{o}(h) .
$$

So, there exists $\bar{r} \in\left(0, r_{0}\right]$ such that $\phi(h, \bar{s})<0$ for every $h \in[0, \bar{r}]$. This proves that, for any $h \in[0, \bar{r}]$, there exists $s(h) \in(0, \bar{s})$ such that

$$
\phi(h, s(h))=\langle\boldsymbol{x}(s(h))-\boldsymbol{y}(h), \mathcal{R} D d(\boldsymbol{y}(h))\rangle=0 .
$$

Furthermore, recalling (52),

$$
0<s(h) \leq \frac{2}{\left\langle\dot{\boldsymbol{x}}(0), e_{1}\right\rangle}[h(1-\tau(x) \kappa(x))+\mathrm{o}(h)] \quad \forall h \in[0, \bar{r}],
$$

so that $s(h) \rightarrow 0$ as $h \downarrow 0$.

Next, observe that, in view of (50), equality (53) can be expressed in intrinsic terms by saying that for any point $y \in \partial \Omega$ of a suitable neighbourhood of $x$, say $U \subset V$, satisfying $\left\langle y-x, e_{1}\right\rangle>0$, there exists $s_{y}:=s\left(h_{y}\right)>0$ such that $\left\langle\boldsymbol{x}\left(s_{y}\right)-y, \mathcal{R} \operatorname{Dd}(y)\right\rangle=0$. Consequently, $\boldsymbol{x}\left(s_{y}\right)=y+\rho_{y} \operatorname{Dd}(y)$ for some $\rho_{y} \in \mathbb{R}$, and 48 will be proved if we show $\rho_{y}>0$. To this end, observe that

$$
h_{y}=|y-x|+\mathrm{o}(|y-x|)
$$


as $\partial \Omega \cap U \ni y \rightarrow x$ satisfying $\left\langle y-x, e_{1}\right\rangle>0$. Also, in view of the above formula and (54),

$$
0<s_{y} \leq C|y-x|
$$

for some constant $C>0$. So, 49 is proved. Furthermore,

$$
\lim _{y \rightarrow x} x\left(s_{y}\right)=\bar{x}=x+\tau(x) \operatorname{Dd}(x),
$$

so that $\rho_{y} \rightarrow \tau(x)$ as $y \rightarrow x$. Hence, $\rho_{y}>0$ for $y$ sufficiently close to $x$, which completes the proof of this step.

Step 3: an estimate for $s_{y}$. We claim that

$$
s_{y}=\frac{1-\tau(x) \kappa(x)}{\left\langle\dot{\boldsymbol{x}}(0), e_{1}\right\rangle}|y-x|+\mathrm{o}(|y-x|)
$$

as $\partial \Omega \cap U \ni y \rightarrow x$ with $\left\langle y-x, e_{1}\right\rangle>0$. Indeed, 51] yields

$$
0=-s_{y}\left\langle\dot{\boldsymbol{x}}(0), e_{1}\right\rangle+h_{y}(1-\tau(x) \kappa(x))+\mathrm{o}\left(h_{y}\right)+\mathrm{o}\left(s_{y}\right) .
$$

The above identity yields the desired result thanks to (55) and (56).

Step 4: an upper bound for $\rho_{y}$. We claim that

$$
\rho_{y} \leq \tau(x)+\frac{1-\tau(x) \kappa(x)}{\left\langle\dot{\boldsymbol{x}}(0), e_{1}\right\rangle}|y-x|+\mathrm{o}(|y-x|)
$$

as $\partial \Omega \cap U \ni y \rightarrow x$ with $\left\langle y-x, e_{1}\right\rangle>0$. Indeed, returning to the parametric representation of $\partial \Omega$ introduced in Step 2, we have, for every $h \in[0, \bar{r}]$,

$$
\begin{aligned}
\rho_{\boldsymbol{y}(h)} & =|\boldsymbol{x}(s(h))-\boldsymbol{y}(h)|=\langle\boldsymbol{x}(s(h))-\boldsymbol{y}(h), D d(\boldsymbol{y}(h))\rangle \\
& =\left\langle\tau(x) e_{2}+s(h) \dot{\boldsymbol{x}}(0)-h e_{1}+\mathrm{o}(h), e_{2}+h D^{2} d(x) e_{1}+\mathrm{o}(h)\right\rangle \\
& =\tau(x)+s(h)\left\langle\dot{\boldsymbol{x}}(0), e_{2}\right\rangle+\mathrm{o}(h)
\end{aligned}
$$

since $0<s(h) \leq C h$. In intrinsic notation, $\rho_{y}=\tau(x)+s_{y}\left\langle\dot{\boldsymbol{x}}(0), e_{2}\right\rangle+\mathrm{o}\left(h_{y}\right)$ for every $y \in \partial \Omega \cap U$ satisfying $\left\langle y-x, e_{1}\right\rangle>0$. Since $\left|\left\langle\dot{\boldsymbol{x}}(0), e_{2}\right\rangle\right| \leq 1$, our claim follows in view of (55) and 57).

Step 5: a global bound. We will now derive the estimate

$$
\frac{1-\tau(x) \kappa(x)}{\left\langle\dot{\boldsymbol{x}}(0), e_{1}\right\rangle} \leq \frac{K}{2}(\operatorname{diam} \Omega)^{2},
$$

which is delicate, since both $\dot{\boldsymbol{x}}(0)=-n_{1}$ and $e_{1}=\mathcal{R}^{-1} D d(x)$ depend on $x$. Let $p_{1}$ and $n_{1}$ be as in Step 1. Then the point $z:=\bar{x}-\tau(x) p_{1}$ belongs to $\Pi(\bar{x})$. Moreover, $\operatorname{Dd}(z)=p_{1}$. So,

$$
z-x=-\tau(x)(D d(z)-D d(x))=\tau(x)\left(e_{2}-p_{1}\right) .
$$


Also,

$$
\left|D d(z)-\left(D d(x)+D^{2} d(x)(z-x)\right)\right| \leq K|z-x|^{2} .
$$

Therefore, recalling that $D^{2} d(x)=-\kappa(x) e_{1} \otimes e_{1}$,

$$
\left|\left(I-\tau(x) \kappa(x) e_{1} \otimes e_{1}\right)\left(p_{1}-e_{2}\right)\right| \leq K \tau^{2}(x)\left|p_{1}-e_{2}\right| \leq \frac{K}{4}(\operatorname{diam} \Omega)^{2}\left|p_{1}-e_{2}\right|^{2} .
$$

Since the matrix $I-\tau(x) \kappa(x) e_{1} \otimes e_{1}$ is positive definite with eigenvalues 1 and $1-$ $\tau(x) \kappa(x)>0$, this proves that

$$
(1-\tau(x) \kappa(x))\left|p_{1}-e_{2}\right| \leq \frac{K}{4}(\operatorname{diam} \Omega)^{2}\left|p_{1}-e_{2}\right|^{2} .
$$

Now, recall that $p_{1} \neq e_{2}$ to conclude

$$
1-\tau(x) \kappa(x) \leq \frac{K}{4}(\operatorname{diam} \Omega)^{2}\left|p_{1}-e_{2}\right| .
$$

Next, the identity $\left\langle\dot{\boldsymbol{x}}(0), p_{1}-e_{2}\right\rangle=0$ implies that $\dot{\boldsymbol{x}}(0)=\lambda \mathcal{R}\left(p_{1}-e_{2}\right)$ for some $\lambda \in \mathbb{R}$ satisfying $|\lambda|=1 /\left|p_{1}-e_{2}\right|$. Therefore,

$$
\left\langle\dot{\boldsymbol{x}}(0), e_{1}\right\rangle=\left|\lambda\left\langle\mathcal{R}\left(p_{1}-e_{2}\right), e_{1}\right\rangle\right|=\frac{\left|\left\langle p_{1}-e_{2}, e_{2}\right\rangle\right|}{\left|p_{1}-e_{2}\right|}=\frac{1-\left\langle p_{1}, e_{2}\right\rangle}{\left|p_{1}-e_{2}\right|} .
$$

Since $\left|p_{1}-e_{2}\right|^{2}=2\left(1-\left\langle p_{1}, e_{2}\right\rangle\right)$, we have $\left\langle\dot{\boldsymbol{x}}(0), e_{1}\right\rangle=\left|p_{1}-e_{2}\right| / 2$. Combining the last equality and (60) proves our claim (59).

Step 6: conclusion. Possibly reducing the neighbourhood $U$ of $x$ that we found in the previous steps, the above construction shows that, for every $y \in U \cap \partial \Omega$ satisfying $\left\langle y-x, e_{1}\right\rangle>0$,

$$
\tau(y) \leq \rho_{y} \leq \tau(x)+K(\operatorname{diam} \Omega)^{2}|y-x| .
$$

By a similar reasoning, there exists another neighbourhood $U^{\prime}$ of $x$ such that, for every $y \in U^{\prime} \cap \partial \Omega$ satisfying $\left\langle y-x, e_{1}\right\rangle<0$,

$$
\tau(y) \leq \tau(x)+K(\operatorname{diam} \Omega)^{2}|y-x| .
$$

Putting these estimates together completes the proof of the lemma.

We are now ready to prove the Lipschitz continuity of $\tau$.

Proof of Theorem 2.12 The conclusion will follow by known results in nonsmooth analysis, once we extend estimate (45) to the $\varepsilon$-neighbourhood $\Omega^{\varepsilon}:=\{x \in \Omega: 0<d(x)<\varepsilon\}$ of $\partial \Omega$. In fact, let $\varepsilon>0$ be such that $d \in \mathcal{C}^{2,1}\left(\Omega^{\varepsilon}\right)$. We claim that there exists a constant $C>0$ so that every $x \in \Omega^{\varepsilon}$ has a ball $B_{\rho}(x) \subset \Omega^{\varepsilon}$ such that

$$
\tau(y) \leq \tau(x)+C|y-x| \quad \forall y \in B_{\rho}(x) .
$$


To show this, observe that for every $y \in \Omega^{\varepsilon}$ such that $\Pi(y)$ is in the neighbourhood $U$ of $\Pi(x)$ provided by Lemma A.1, we have, in view of 45,

$$
\begin{aligned}
\tau(y) & =\tau(\Pi(y))-d(y) \\
& \leq \tau(\Pi(x))+K(\operatorname{diam} \Omega)^{2}|\Pi(y)-\Pi(x)|-d(y) \\
& \leq \tau(x)+K(\operatorname{diam} \Omega)^{2}\|D \Pi\|_{\infty, \Omega^{\varepsilon}}|y-x|+d(x)-d(y) .
\end{aligned}
$$

Our claim (61) follows with $C=K(\operatorname{diam} \Omega)^{2}\|D \Pi\|_{\infty, \Omega^{\varepsilon}}+1$.

Next, we will derive the bound

$$
|p| \leq C \quad \forall p \in \partial_{P} \tau(x) \forall x \in \Omega^{\varepsilon},
$$

where $\partial_{P} \tau(x)$ denotes the proximal subgradient of $\tau$ at $x$ and $C$ is the constant that appears in (61). Then, by [13, Theorem 7.3, p. 52], such an estimate will imply that $\tau$ is Lipschitz in $\Omega^{\varepsilon}$, and so on $\partial \Omega$ as well. To check $(63)$, recall that a vector $p \in \mathbb{R}^{2}$ belongs to $\partial_{P} \tau(x)$ if and only if there exist numbers $\sigma, \eta>0$ such that

$$
\tau(y) \geq \tau(x)+\langle p, y-x\rangle-\sigma|y-x|^{2} \quad \forall y \in B_{\eta}(x),
$$

by [13, Theorem 2.5, p. 33]. Now, combine the above inequality with (61) to obtain

$$
\langle p, y-x\rangle \leq C|y-x|+\sigma|y-x|^{2}
$$

whenever $|y-x|<\min \{\rho, \eta\}$. This implies (63) and completes the proof.

Acknowledgements. The authors are grateful to Italo Capuzzo-Dolcetta for attracting their attention to reference [22].

\section{References}

[1] Albano, P., Cannarsa, P.: Structural properties of singularities of semiconcave functions. Ann. Scuola Norm. Sup. Pisa Cl. Sci. 28, 719-740 (1999) Zbl 0957.26002 MR 2001g:26011

[2] Ambrosio, L.: Optimal transport maps in Monge-Kantorovich problem. In: Proc. Internat. Congress of Mathematicians, Vol. III (Beijing 2002), Higher Ed. Press, Beijing, 131-140 (2002) Zbl 1005.49030 MR 2004c:49087

[3] Ambrosio, L., Cannarsa, P., Soner, H. M.: On the propagation of singularities of semi-convex functions. Ann. Scuola Norm. Sup. Pisa Cl. Sci. (4) 20, 597-616 (1993) Zbl 0874.49041 MR 95b:49068

[4] Aronsson, G.: A mathematical model in sand mechanics: Presentation and analysis. SIAM J. Appl. Math. 22, 437-458 (1972) Zbl 0242.34048 MR 46 \#7641

[5] Aronsson, G., Evans, L. C.: An asymptotic model for compression molding. Indiana Univ. Math. J. 51, 1-36 (2002) Zbl pre01780931 MR 2003d:76053

[6] Aronsson, G., Evans, L. C., Wu, Y.: Fast/slow diffusion and growing sandpiles. J. Differential Equations 131, 304-335 (1996) Zbl 0864.35057 MR 97i:35068

[7] Bardi, M., Capuzzo-Dolcetta, I.: Optimal Control and Viscosity Solutions of HamiltonJacobi-Bellman Equations. Systems and Control: Foundations and Applications, Birkhäuser, Boston (1997) Zbl 0890.49011 MR 99e:49001 
[8] Bouchitté, G., Buttazzo, G., Seppechere, P.: Shape optimization solutions via MongeKantorovich equation. C. R. Acad. Sci. Paris Sér. I Math. 324, 1185-1191 (1997) Zbl 0884.49023 MR 98i:49044

[9] Bouchitté, G., Buttazzo, G.: Characterization of optimal shapes and masses through Monge-Kantorovich equation. J. Eur. Math. Soc. 3, 139-168 (2001) Zbl 0982.49025 MR 2002c:49080

[10] Boutreux, T., de Gennes, P.-G.: Surface flows of granular mixtures, I. General principles and minimal model. J. Phys. I France 6, 1295-1304 (1996)

[11] Bhattacharya, T., Di Benedetto, E., Manfredi, J.: Limits as $p \rightarrow \infty$ of $\Delta_{p} u_{p}=f$ and related extremal problems. In: Some Topics in Nonlinear PDEs (Turin, 1989), Rend. Sem. Mat. Univ. Politec. Torino 1989, Special Issue, 15-68 (1991) MR 93a:35049

[12] Cellina, A., Perrotta, S.: On the validity of the maximum principle and of the Euler-Lagrange equation for a minimum problem depending on the gradient. SIAM J. Control Optim. 36, 1987-1998 (1998) Zbl 0926.49014 MR 99g:4901

[13] Clarke, F. H., Ledyaev, Yu. S., Stern, R. J., Wolenski, P. R.: Nonsmooth Analysis and Control Theory. Springer, New York (1998) Zbl pre01113627 MR 99a:49001

[14] Erdős, P.: Some remarks on the measurability of certain sets. Bull. Amer. Math. Soc. 51, 728-731 (1945) Zbl 0063.01269 MR 7,197f

[15] Evans, L. C., Feldman, M., Gariepy, R.: Fast/slow diffusion and collapsing sandpiles. J. Differential Equations 137, 166-209 (1997) Zbl 0879.35019 MR 98g:35093

[16] Evans, L. C., Gangbo, W.: Differential equations methods for the Monge-Kantorovich mass transfer problem. Mem. Amer. Math. Soc. 137, no. 653 (1999) Zbl 0920.49004 MR 99g:3513

[17] Evans, L. C., Gariepy, R. F.: Measure Theory and Fine Properties of Functions. Stud. Adv. Math., CRC Press, Boca Raton, FL (1992) Zbl 0804.28001 MR 93f:28001

[18] Feldman, M.: Variational evolution problems and nonlocal geometric motion. Arch. Rat. Mech. Anal. 146, 221-274 (1999) Zbl 0955.49025 MR 2000h:35066

[19] Feldman, M.: Growth of a sandpile around an obstacle. In: Monge Ampère Equation: Appplications to Geometry and Optimization (Deefield Beach, FL, 1997), Contemp. Math. 226, Amer. Math. Soc., Providence, RI, 55-78 (1999) Zbl 0924.35176 MR 2000c:49066

[20] Feldman, M., McCann, R. J.: Uniqueness and transport density in Monge's mass transportation problem. Calc. Var. 15, 81-113 (2002) Zbl 1003.49031 MR 2003e:49068

[21] Gilbarg, D., Trudinger, N. S.: Elliptic Partial Differential Equations of Second Order. Grundlehren Math. Wiss. 224, Springer, Berlin (1983)

[22] Hadeler, K. P., Kuttler, C.: Dynamical models for granular matter. Granular Matter 2, 9-18 (1999)

[23] Itoh, J. , Tanaka, M.: The Lipschitz continuity of the distance function to the cut locus. Trans. Amer. Math. Soc. 353, 21-40 (2001) Zbl 0971.53031 MR 2001b:53029

[24] Janfalk, U.: Behaviour in the limit, as $p \rightarrow \infty$, of minimizers of functionals involving $p$-Dirichlet integrals. SIAM J. Math. Anal. 27, 341-360 (1996) Zbl 0853.35028 MR 96m:49023

[25] Li, Y. Y., Nirenberg, L.: The distance function to the boundary, Finsler geometry and the singular set of viscosity solutions of some Hamilton-Jacobi equations. Preprint (2003)

[26] Motzkin, Th.: Sur quelques propriétés caractéristiques des ensembles convexes. Atti Acad. Naz. Lincei Rend. Cl. Sci. Fis. Mat. Natur. 21, 562-567 (1935) Zbl 0011.41105

[27] Prigozhin, L.: Variational model of sandpile growth. European J. Appl. Math. 7, 225-235 (1996) Zbl 0913.73079 MR 97j:73065

[28] Rockafellar, R. T., Wets, R. J.-B.: Variational Analysis. Grundlehren Math. Wiss. 317, Springer, Berlin (1998) Zbl 0888.49001 MR 98m:49001 